\title{
A dynamical mechanism for large volumes with consistent couplings
}

\author{
Steven Abel \\ IPPP, Durham University, \\ Durham, DH1 3LE, U.K. \\ E-mail: s.a.abel@durham.ac.uk
}

ABSTRACT: A mechanism for addressing the "decompactification problem" is proposed, which consists of balancing the vacuum energy in Scherk-Schwarzed theories against contributions coming from non-perturbative physics. Universality of threshold corrections ensures that, in such situations, the stable minimum will have consistent gauge couplings for any gauge group that shares the same $\mathcal{N}=2$ beta function for the bulk excitations as the gauge group that takes part in the minimisation. Scherk-Schwarz compactification from $6 \mathrm{D}$ to $4 \mathrm{D}$ in heterotic strings is discussed explicitly, together with two alternative possibilities for the non-perturbative physics, namely metastable SQCD vacua and a single gaugino condensate. In the former case, it is shown that modular symmetries gives various consistency checks, and allow one to follow soft-terms, playing a similar role to $R$-symmetry in global SQCD. The latter case is particularly attractive when there is nett Bose-Fermi degeneracy in the massless sector. In such cases, because the original Casimir energy is generated entirely by excited and/or non-physical string modes, it is completely immune to the non-perturbative IR physics. Such a separation between UV and IR contributions to the potential greatly simplifies the analysis of stabilisation, and is a general possibility that has not been considered before.

KEYwords: Superstring Vacua, Superstrings and Heterotic Strings

ArXiv EPrint: 1609.01311 


\section{Contents}

1 Introduction 1

2 The mechanism in a 5D non-maximal Scherk-Schwarz model 4

$\begin{array}{ll}2.1 & \text { The generic Casimir energy case }\end{array}$

2.2 The exponentially suppressed (UV-Casimir energy) case 9

3 String/supergravity embedding $\quad 11$

$\begin{array}{lll}3.1 & \text { Spectrum and congruence subgroups in the effective supergravity theory } & 12\end{array}$

$\begin{array}{ll}3.2 & \text { Calculation of Casimir energy } \\ & 18\end{array}$

3.3 The congruence subgroup method for mapping soft-terms between Seiberg duals 21

$\begin{array}{lll}3.4 & \text { On } \Lambda_{e}>M_{K K} & 25\end{array}$

4 Stabilisation in the string-embedded theories $\quad 26$

$\begin{array}{lll}4.1 & \text { Generic Casimir energy } 26\end{array}$

4.2 UV-Casimir energy balanced against a gaugino condensate 29

5 Conclusion $\quad 31$

A Summary of $\operatorname{SL}(2, \mathbb{Z})_{U}$ and $\operatorname{SL}(2, \mathbb{Z})_{U}$ structure 32

\section{Introduction}

The Scherk-Schwarz (SS) mechanism is one of the most attractive means of spontaneously breaking supersymmetry (SSSB) [1,2]. In the SS mechanism, supersymmetry is broken by compactification with $R$-symmetry violating boundary conditions, and from a $4 \mathrm{D}$ perspective the inverse volume $1 / R^{d}$ (where $R$ is used as a generic compactification scale) plays the role of an order parameter for supersymmetry breaking in the effective field theory. This yields all-orders control over supersymmetry breaking, and shields dimensionful operators such as the Casimir energy and soft-terms from the ultra-violet (UV) completion [3-7]. They can then largely be computed as finite Kaluza-Klein (KK) contributions in an effective extra-dimensional field theory, enhancing predictivity. There are numerous interesting phenomenological applications, for example in the recent work of refs. [8-14].

In such theories, a volume significantly larger than the fundamental scale, $R \gg \ell_{s}$, is necessary (even if one does not insist on low scale supersymmetry breaking) if one wishes the reproduce the physics of the traditional field theory SS mechanism. This is because heavy modes come to dominate over the KK modes in loop processes once the compactification radius approaches the fundamental length scale (see the discussion in 
ref. [15]). In the context of non-supersymmetric string theory for example, "non-physical" proto-gravitons start to be important once $R \lesssim 2 \ell_{s}$. The necessary separation between the UV completion and the KK scale can be achieved by configurations that interpolate from supersymmetric theories at large radius to non-supersymmetric ones at small radius [15]. Ideally, one would then like to treat this as an approximate "moduli space", and generate a consistent supersymmetry breaking solution at large volume dynamically. This has been widely discussed in the Scherk-Schwarz context in for example refs. [16-24].

However large volumes are problematic in the context of heterotic string theory. They are felt universally by the gauge couplings, which are then generally rendered inconsistent at one-loop by the corresponding KK mode contributions. This is a generic source of tension for the SS mechanism in heterotic strings and indeed any SS set-up that does not have a "brane" configuration.

To be specific, consider an effective 5D SQCD theory (i.e. one in which only one compactified dimension is significantly larger than the fundamental scale). Supposing that any other moduli except the radius are already stabilised at small volumes (so they play no further role in the dynamics or in the magnitude of the gauge couplings) the expression for the gauge coupling of the effective $4 \mathrm{D}$ SQCD theory is

$$
\frac{16 \pi^{2}}{g^{2}(\mu)}=k \frac{16 \pi^{2}}{g_{s}^{2}}+b \ln \frac{M_{s}^{2}}{\mu^{2}}+\Delta(R),
$$

where $b$ is the beta function coefficient of the original effective $4 \mathrm{D} \mathcal{N}=1$ theory (in a convention where $\mathrm{SU}(N)$ supersymmetric QCD with $F$ flavours would have $b=-3 N+F$ ), and $\Delta$ are the offending threshold contributions which at large volumes are dominated by the KK sector of the theory,

$$
\Delta(R)=C R M_{s}-2 b \ln \left(R M_{s}\right) .
$$

The constant $C$ depends on various other parameters and moduli, most importantly on the beta functions of the $\mathcal{N}=2$ content of the theory. In this preliminary discussion (and in fact right up to the last section) $g_{s}$ will be assumed to be fixed beforehand: ultimately though it will also be dynamical, being given by the VEV of the axio-dilaton.

There are then two possibilities assuming that $C \neq 0$. Gauge couplings that have $C>0$ are made weaker by the threshold corrections. Broadly speaking one can interpret this as the contribution from power-law running between the fundamental scale and the KK scale [25-29] (although there are various subtleties in mapping extra-dimensional fieldtheory to string theory - see for example ref. [7]). At large volume the couplings become tiny and the corresponding symmetry is to all intents and purposes global. By contrast those couplings that have $C<0$ grow stronger at large radius, from extremely weak values at the fundamental scale. They can in principle become reasonably large, but then one has to balance the threshold contribution to $1 / g^{2}$ against its tree-level value. It should be noted that $C$ and $b$ need not have the same sign, so there is nothing to prevent a theory flowing to stronger coupling at the KK scale, and then for the effective $4 \mathrm{D}$ theory to be IR-free (and vice-versa); to simplify the discussion it will be assumed that they do have the same sign. 
To summarise the difficulty, $C>0$ couplings are insignificant at low energy unless the gauge symmetry is localized in the large volume, implying some kind of brane set-up. On the other hand, $C<0$ couplings seem to imply a fine-tuning of tree-level against radiative corrections, so that they are extremely weak at the string scale, but order one just at the bottom of the KK tower where they enter the logarithmically running $4 \mathrm{D}$ regime. This issue, which has become known as the "decompactification problem", has been discussed in the past in for example in refs. [28-36], and was eloquently summarized recently in ref. [37]. Special theories are known that circumvent the coupling/volume sensitivity because they do have $C=0$ [34-37], but here it will be of interest to consider more generic models.

The purpose of this paper is to argue that there is in fact a way to realise order one couplings at large volume dynamically and without fine-tuning, providing a solution to the decompactification problem for a much broader class of models. The set-up is very general: it requires only that the compact volume is stabilised by balancing a dynamical transmutation scale, $\Lambda_{e}$, against a leading order one-loop Casimir energy. This results in a gauge coupling that is inevitably becoming large precisely where the volume is stabilised. The particular gauge factor that takes part in the stabilisation may of course be of little further use for phenomenology, depending on the precise non-perturbative physics behind the appearance of $\Lambda_{e}$. However the universality in the gauge couplings and their $\mathcal{N}=2$ threshold corrections ensures that any gauge group with the same $C$ will also have gauge couplings of order one, with only logarithmic differences appearing due to the different $\mathcal{N}=1$ beta functions, $b$. (Note the gauge group and particle content do not have to be the same, so for example the content of an $\mathcal{N}=2 \mathrm{SU}(5)$ SQCD with 6 flavours has a $C$ equal to that of $\mathcal{N}=2 \mathrm{SU}(3) \mathrm{SQCD}$ with 2 flavours.) That such universality exists even in theories that have supersymmetry broken by the Scherk-Schwarz mechanism has been recently shown in ref. [38]. Meanwhile those gauge factors with larger or smaller $C$ will become effectively global or strongly coupled and confined, respectively, and will play little further role in phenomenology.

The configuration that will be studied here is based on the interplay of two competing mildly repulsive and mildly attractive effects. The first is the aforementioned Casimir energy that arises in compactifications where supersymmetry is spontaneously broken by the SS mechanism. This typically goes as $\left(N_{f}^{0}-N_{b}^{0}\right) / R^{4}$, where $R$ is the compactification scale along the direction that breaks supersymmetry, and $\left(N_{f}^{0}-N_{b}^{0}\right)$ is the nett FermiBose number of the states left massless by the SS mechanism; choosing it to be positive, it represents a repulsive effect running away to large radius. The competing effect is a positive contribution to the cosmological constant arising from some non-perturbative process. We will consider two options: the first is an SQCD sub-sector of the theory which sits in the metastable supersymmetry breaking minimum of Intriligator, Seiberg and Shih [39] (ISS) and the second is a Yang-Mills gaugino condensate. Both of these produce terms that are governed by the dynamical scale of the theory, which in turn depends on the threshold contribution to the effective gauge coupling in eq. (1.1). Assuming that both $C$ and $b$ are negative, this contribution increases with radius, so it is attractive.

The result is that the theory is driven dynamically to the boundary of the perturbative moduli space and minimised there, with all gauge couplings that share the same value of $C$ 
automatically taking values of order one no matter how small the (universal) string-scale value. It is clear that the resulting large volume is then directly related to the smallness of the string-scale coupling at the origin.

The next section presents a 5D toy-version of the mechanism, expressed purely in field theory. It emphasises the general difference between an SS vacuum energy that is broadly the same as the field theoretical one described above, and the qualitatively different possibility that heavy UV modes in the theory dominate the SS vacuum energy. This may simply be a result of the volume approaching the string scale, in which case (as mentioned above) the leading contributions come from non-physical modes, or it may be a result of the massless contributions vanishing in theories that have $\left(N_{f}^{0}=N_{b}^{0}\right)$, in which case the leading contributions come from the lowest lying string excitations. In these cases the SS vacuum energy cannot be well understood in extra-dimensional field theory, but can be easily calculated in string theory. Moreover an important and recurring theme is that, because it is UV in nature, the SS vacuum energy in such cases is completely immune to any non-perturbative physics that one might balance it against in order to produce a stable compactification. In order to emphasise the distinction, this kind of SS induced vacuum energy will be referred to as UV-Casimir energy.

Section 3 collects the necessary ingredients required for the string realisation. One of the reasons for interest in the ISS mechanism in this context rather than just gaugino condensation will become clear: it allows several checks of the stringy implementation of non-perturbative supersymmetry breaking, and in the generic SS case it gives a cleaner separation between the contributions to the potential coming from the SS and ISS mechanisms. The Casimir energy is calculated in toroidal SS compactifications from 6D to $4 \mathrm{D}$, the residual modular symmetry is discussed and several new results are presented, on the use of modular invariance to follow the SS induced soft terms, and on a consistency condition for the stringy implementation of the ISS mechanism.

These results are used section 4 to study stabilisation for generic Casimir energies, and also for the case in which an exponentially suppressed UV-Casimir balances against a gaugino condensate. Up to this point, the approach is somewhat modular in that the tree-level coupling $g_{s}$ and also its axionic partner are taken to be fixed parameters in order to investigate how the compactification dynamics adjusts to consistently accommodate tiny values. In this last example all moduli $(S, T, U)$ are treated as dynamical fields. The beauty of UV-Casimir energy becomes evident here, and it is worth repeating it: because it is blind to IR physics, one can essentially balance two robustly independent contributions to the vacuum energy that are nevertheless functions of only the three $S, T, U$ moduli. An additional interesting feature here is that the gaugino condensate scale automatically adjusts to roughly match that of the UV-Casimir energy.

\section{The mechanism in a 5D non-maximal Scherk-Schwarz model}

It is convenient to proceed by developing the 5D example of the mechanism outlined in the Introduction, with the non-perturbative physics being the ISS mechanism. Although it illustrates the principle, it should be regarded as something of a warm-up exercise to the 
more stringy implementation in forthcoming sections. In particular, an important question is whether the soft-terms induced by the SS mechanism can disrupt the supersymmetry breaking of the ISS mechanism, which is after all written entirely within $\mathcal{N}=1$ supersymmetric QCD. In the next section, we shall learn how to treat this question by mapping soft-terms using the modular symmetry of the $6 D \rightarrow 4 D$ compactification. There we will also consider gaugino condensation as an alternative non-perturbative mechanism. For the moment we shall solve this issue by invoking non-maximal SS phases.

It will be sufficient to assume that the Scherk-Schwarz action shifts the masses of vector-like pairs of states. (It could also act on chiral states but it would not qualitatively change the discussion.) The KK masses take the form $\left(n+q_{F \pm}\right) / R$, and $\left(n+q_{B \pm}\right) / R$, where $q_{B \pm}=\left( \pm \alpha_{B}+R m_{D}\right)$ and $q_{F \pm}=\left( \pm \alpha_{F}+R m_{D}\right)$, and where $m_{D}$ is an elementary supersymmetric Dirac mass (a.k.a. $\mu$-term).

There are limitations as to where the mechanism can work in its most naive form. As mentioned above the main constraint arises from the fact that the results of ISS are derived in $4 \mathrm{D} \mathcal{N}=1$ supersymmetric QCD, whereas this is a $5 \mathrm{D}$ setting in which supersymmetry is already partially broken by the SS mechanism. If one wishes to adopt the ISS results at face-value (with no extra KK modes to complicate things), one can impose a modest energy gap between the dynamical scale of the the SQCD theory and the mass-scale of the lowest lying KK modes, and in addition between the two sources of superymmetry breaking to ensure that the ISS analysis is not disrupted by the soft-terms that are already induced by the SS mechanism. The latter are expected to remain of order $\alpha_{F, B} / R$ throughout (in both the electric and magnetic SQCD phases), so the ISS results can be used wholesale if this scale is much less than the supersymmetry breaking induced in the low energy theory of the ISS mechanism. This can be achieved by assuming non-maximal Scherk-Schwarz phases, $\alpha_{F, B} \ll 1 / 2$. Such non-maximal phases are somewhat artificial in the stringy Scherk-Schwarz mechanism [17, 22, 40-47] because $\alpha_{F, B}$ are proportional to some linear combination of gauge and $R$-charges and can only take discrete values. In some orbifold compactifications, these could be for example $1 / 5$, but they cannot be arbitrarily small. As mentioned, a more realistic implementation will ultimately require a proper treatment of the mapping of soft-terms in the SS context, including KK modes, and a properly adjusted ISS picture to take account of them.

The last constraint is on the elementary supersymmetric Dirac mass required in the ISS mechanism: it should take values $m_{D} \ll 1 / R$. It is simple and natural - although not crucial - to take $m_{D}$ also to be induced by the compactification, so that it too is proportional to $1 / R$, with constant of proportionality $\alpha_{D}=R m_{D} \ll 1$. In this $5 \mathrm{D}$ model therefore, we shall maintain the following hierarchy of scales:

$$
\frac{1}{R} \gtrsim \Lambda_{e} \gg \sqrt{\Lambda_{e} \alpha_{D} / R} \gg \frac{\alpha_{F, B}}{R} .
$$

The left-most scale is the bottom of the KK tower, which is taken to be greater than the dynamical scale $\Lambda_{e}(R)$ of the effective 4D SQCD theory. Meanwhile $m_{D}$ must be smaller than $\Lambda_{e}(R)$ so that states which get a Dirac mass are not simply integrated out. And finally, on the right, a sufficient condition for the $4 \mathrm{D} \mathcal{N}=1$ ISS analysis to be a 
good approximation, is that the scale of effective supersymmetry breaking induced by the Scherk-Schwarz mechanism is negligible compared to the supersymmetry breaking induced later by the ISS mechanism. These constraints translate into a condition on $\Lambda_{e} R$ of

$$
1 \gtrsim R \Lambda_{e} \gg \alpha_{D}, \frac{\alpha_{F, B}^{2}}{\alpha_{D}}
$$

It will be convenient to assume $\alpha_{D} \sim \alpha_{F, B}$.

\subsection{The generic Casimir energy case}

The potential may now be determined, beginning with the Casimir contribution. For definiteness let us take $N_{b}^{0}$ of the $\alpha_{B}$ and $N_{f}^{0}$ of the $\alpha_{F}$ to be exactly zero, and the rest to be degenerate with $\alpha_{B}=\alpha_{F}=\alpha \ll 1$. The light theory then has $N_{f}^{0}$ massless fermions and $N_{b}^{0}$ massless bosons, with the remainder having mass $\sim \alpha / R$. The one-loop Casimir energy can be computed at the level of the 5D KK theory regardless of any more fundamental UV completion, because it is dominated by the massless modes and their KK excitations (assuming that the KK levels do not have equal numbers of fermions and bosons). The simplest method is to Poisson resum the Schwinger integral form of the Coleman-Weinberg potential;

$$
\begin{array}{r}
V_{C}=-\frac{1}{16 \pi^{2}} \operatorname{Tr} \sum_{n} \int_{0}^{\infty} \frac{d t}{t^{3}} \exp \left[-t\left(n+q_{B+}\right)^{2} / R^{2}\right]+\exp \left[-t\left(n+q_{B-}\right)^{2} / R^{2}\right] \\
-\exp \left[-t\left(n+q_{F+}\right)^{2} / R^{2}\right]-\exp \left[-t\left(n+q_{F-}\right)^{2} / R^{2}\right]
\end{array}
$$

where the trace is over the supermultiplet representations. The insensitivity of the Casimir energy to the UV-completion is evident here in the fact that there is no need for a UV cut-off on the integral. (In other words a full string calculation as in ref. [15] would just give additional exponentially suppressed corrections.) Poisson resumming this expression gives

$$
\begin{array}{r}
V_{C}=-\frac{1}{16 \pi^{2}} \operatorname{Tr} \int_{0}^{\infty} d t R \pi^{1 / 2} t^{-7 / 2} \sum_{\ell=-\infty}^{\infty} e^{-\ell^{2} \pi^{2} R^{2} / t}\left[\cos \left(2 \pi \ell q_{B+}\right)+\cos \left(2 \pi \ell q_{B-}\right)\right. \\
\left.-\cos \left(2 \pi \ell q_{F+}\right)-\cos \left(2 \pi \ell q_{F-}\right)\right],
\end{array}
$$

and performing the integral gives

$$
V_{C}=\operatorname{Tr}\left[B\left(q_{F+}\right)+B\left(q_{F-}\right)-B\left(q_{B+}\right)-B\left(q_{B-}\right)\right],
$$

where (in agreement with e.g. $[5,7,9,11,17])$

$$
B(x)=\frac{3}{64 \pi^{6} R^{4}}\left(\mathrm{Li}_{5} e^{2 \pi i x}+\mathrm{Li}_{5} e^{-2 \pi i x}\right) .
$$

Expanding in the $\alpha$ 's gives,

$$
V_{C}=\frac{3 \zeta(3)}{8 \pi^{4}} \frac{\left(N_{f}^{0}-N_{b}^{0}\right) \alpha^{2}}{R^{4}}
$$


The second ingredient for the potential is of course the ISS contribution from an $S Q C D$ sector. Assuming that the original theory contains an $\mathrm{SU}(N)$ gauge group with $F$ flavours of fundamental/antifundamental pairs of chiral superfields, the potential comes from the O'Raighfeartaigh superpotential of the magnetic SQCD theory, and takes the form

$$
W_{I S S}=h \operatorname{Tr}(q \Phi \tilde{q})-\frac{\alpha_{D} \Lambda_{e}}{R} \operatorname{Tr}(\Phi),
$$

where $q, \tilde{q}$ are magnetic quarks, $\Phi$ is the $F \times F$ bound state meson, and where ignorance about the precise normalization of $\Phi$ has been absorbed into the parameters $\alpha_{D}$ and $h .{ }^{1}$

Provided that the number of colours and flavours is such that the SQCD theory is in the free magnetic window, $N+1<F \leq 3 N / 2$, the result is an additional tree-level term in the potential of the form

$$
V_{I S S}=N \alpha_{D}^{2}\left(\frac{\Lambda_{e}}{R}\right)^{2}
$$

The total potential is

$$
V=R^{-4}\left[\alpha^{2} \rho\left(N_{f}^{0}-N_{b}^{0}\right)+\alpha_{D}^{2} N\left(R \Lambda_{e}\right)^{2}\right],
$$

where $\rho=\frac{3 \zeta(3)}{8 \pi^{4}} \approx 5 \times 10^{-3} \ll 1$.

As an aside, note that for negative Casimir energy the potential can be precisely zero while still satisfying the conditions in eq. (2.2) for the $\mathcal{N}=1$ supersymmetric ISS analysis to be valid: indeed a zero potential requires only

$$
\left(R \Lambda_{e}\right)^{2}=\frac{\left(N_{b}^{0}-N_{f}^{0}\right)}{N} \rho \frac{\alpha^{2}}{\alpha_{D}^{2}} .
$$

Conversely, for positive Casimir energy, one may now invoke the $R$-dependence of $\Lambda_{e}$ using eq. (1.1) to minimize the potential. Taking $k=1$ and setting the dynamical scale of the $S Q C D$ theory to be where $1 / g^{2}\left(\Lambda_{e}\right)=0$ gives

$$
\left(R \Lambda_{e}\right)^{2}=e^{-\frac{16 \pi^{2}}{g_{s}^{2}|b|}+\frac{C}{b} R M_{s}} .
$$

It is convenient to define a fiducial coupling $g_{0}$ (which is of order $g_{s}$ ), and a corresponding fiducial scale, $\mu_{0}$, given by

$$
\begin{aligned}
\frac{16 \pi^{2}}{g_{0}^{2}} & =\frac{16 \pi^{2}}{g_{s}^{2}}+b \ln \frac{M_{s}^{2}}{\mu_{0}^{2}} \\
\frac{\mu_{0}^{2}}{M_{s}^{2}} & =\frac{\alpha^{2} \rho\left(N_{f}^{0}-N_{b}^{0}\right)}{\alpha_{D}^{2} N} \sim 10^{-2} .
\end{aligned}
$$

\footnotetext{
${ }^{1}$ More precisely, following ref. [39], if the original SQCD theory has a dynamical scale $\Lambda_{e}$, a superpotential $W_{e}=m_{D} Q \tilde{Q}$, and a canonically normalized meson $\hat{\Phi}=\gamma^{-1} Q \tilde{Q} / \Lambda_{e}$, then $W_{I S S} \equiv \sqrt{\gamma} h \operatorname{Tr}(q \hat{\Phi} \tilde{q})-$ $\sqrt{\gamma} m_{D} \Lambda_{e} \operatorname{Tr}(\hat{\Phi})$, with the understanding that $W_{I S S}$ is to be treated as a global superpotential. This issue will become important later and will be revisited, when a proper distinction between the physical and holomorphic scales will be made.
} 
The full potential has a minimum at

$$
\begin{aligned}
R_{\min } M_{s} & =\frac{b}{C}\left[4+W\left(4 e^{-4} \frac{\mu_{0}^{2}}{M_{s}^{2}} e^{\frac{16 \pi^{2}}{|b| g_{s}^{2}}}\right)\right] \\
& =\frac{1}{|C|} \frac{16 \pi^{2}}{g_{0}^{2}}+\mathcal{O}(1)
\end{aligned}
$$

where $W$ is the Lambert $W$-function (a.k.a. product log). Eq. (2.13) then gives

$$
R_{\min } \Lambda_{e}=\mu_{0} / M_{s}
$$

If the parameters are all of similar magnitude, $\left(N_{f}^{0}-N_{b}^{0}\right) / N_{c} \sim \alpha_{D} / \alpha \sim 1$, then eq. (2.13) gives $R_{\min } \Lambda_{e} \sim \sqrt{C} \approx 0.07$, automatically satisfying the requirement in eq. (2.2) and achieving the desired effect of the QCD theory ending up with a dynamical scale somewhat below the KK mass-scale, $M_{K K}=1 / R_{\min }$, even if $16 \pi^{2} / g_{s}^{2} \sim R_{\min } M_{s}$ is chosen to be huge. In order to satisfy the other constraints of eq. (2.2), under the assumption that $\alpha_{D} \sim \alpha_{F, B}=\alpha$ one requires only that $\alpha^{2} \ll \rho \frac{\left(N_{f}^{0}-N_{b}^{0}\right)}{N}$ which is relatively easy to achieve. (For example with $N=N_{f}^{0}-N_{b}^{0}$, one requires $\alpha \lesssim 1 / 10$ which is conceivably possible even within some string orbifold models). The value of the cosmological constant at the minimum is given by

$$
\begin{aligned}
V\left(R_{\min }\right) & =R_{\min }^{-4}\left[2 \rho \alpha^{2}\left(N_{f}^{0}-N_{b}^{0}\right)\right] \\
& \approx \frac{g_{s}^{8} M_{s}^{4}}{\left(16 \pi^{2}\right)^{4}}\left[2 \rho \alpha^{2}\left(N_{f}^{0}-N_{b}^{0}\right)\right] .
\end{aligned}
$$

As promised the minimum is automatically balanced to appear at the correct values of $R_{\text {min }}$. An example of the potential is shown in figure 1 for sample values. It is essentially a $1 / R^{4}$ runaway to large radius until the ISS contribution takes over where the SQCD gauge coupling is starting to become strong. The minimum is de Sitter, and of order $10^{-3} M_{K K}^{4}$. Clearly for consistency one would then require some additional $R$-independent and negative contribution to bring the final cosmological constant close to zero.

Note that going along implicitly with need to protect the ISS mechanism from the supersymmetry breaking of the SS mechanism, is of course the converse assumption that the effects of strong coupling in the SCQD sector do not disrupt the original calculation of the Casimir energy. This assumption is credible because the latter is dominated by the tower of KK states with masses between $M_{K K}$ and $M_{s}$, and above physics occurring at the scale $\Lambda_{e}$ provided that the $\Lambda_{e}<1 / R_{\min }$ constraint is satisfied. This condition can be relaxed in various cases and under various assumptions which will be made more precise when we come to study the string embedding in later sections.

The form of the potential for $R \gg R_{\min }$ is not well determined. In these regions the dynamical scale is larger than the KK scale (i.e. $R \Lambda_{e}(R) \gg 1$ ) so the sufficient condition in eq. (2.1) is violated. Most probably this implies that the potential turns over at some point, and the minimum at $R_{\min }$ is metastable in the $R$ direction as well, with larger values simply reverting to runaway behaviour. It is not clear how the large radius limit of such 
theories lifts to the decompactified 6D theory; most likely it is related to the 4D IR free magnetic dual of the ISS theory, rather than the original electric SQCD theory.

If one makes the conservative assumption that the minimum derived above is indeed only metastable, it is important to consider what the tunnelling rate would be to continued runaway along $R$, in order to confirm that it is sufficiently small. An estimate requires the normalization of the modulus corresponding to $R$. In flat space compactifications derived from string theory the Kähler potential is given by $K \sim-\log V$ where $V$ is the overall compactification volume. In the present case one can identify $V \sim i\left(T_{R}-\bar{T}_{R}\right)$ with $T_{R}$ being a holomorphic modulus whose imaginary part gives $R$. This would give kinetic terms for $R$ of the form $\mathcal{L} \supset \frac{\left|\partial T_{R}\right|^{2}}{R^{2}}$ so the canonically normalised field is $\phi_{R}=T_{R} / R_{\min }$. The tunnelling action can then be approximated in the thick wall limit. The advantage of this physical situation is that the height of the barrier does not appear in the action at leading order, only its width and the difference $\Delta V$ between the vacuum energies of the false and true minima. A crude estimate for the action is then [48]

$$
S_{E} \sim 2 \pi^{2} \frac{\left(\Delta \phi_{R}\right)^{4}}{\Delta V}
$$

where $\Delta V=V_{\text {false }}-V_{\text {true }}=R_{\min }^{-4} \alpha_{D}^{2} N\left(R_{\min } \Lambda_{e}\right)^{2}$. As this is a sufficient condition, let us adopt a conservative value for $\Delta \phi_{R}$, namely the distance in field-space between $R_{\min }$ and the point where perturbativity breaks down, $\Lambda_{e} R \sim 1$, or $\frac{\Lambda_{e} R_{\min }}{\Lambda_{e} R}=\mu_{0} / M_{s}=e^{-\frac{C}{b} \Delta R M_{s}}$. This gives

$$
\Delta \phi_{R} \approx R_{\min }^{-1} \frac{b}{C} \log \frac{M_{s}}{\mu_{0}},
$$

leading to an estimate for the tunnelling action of

$$
S_{E} \sim \frac{2 \pi^{2}}{\alpha^{2}}\left(\frac{M_{s}}{\mu_{0}}\right)^{2} \sim 10^{3} / \alpha^{2}
$$

This is well above the $S_{E} \gtrsim 400$ that is required to ensure stability on timescales of the age of the universe (see e.g. ref. [49] and references therein). Heuristically this is simply a consequence of the fact that the Casimir energy in $V$ is a one-loop effect (given by $\rho$ ), so the potential is much flatter than it is broad.

\subsection{The exponentially suppressed ( $U V$-Casimir energy) case}

Models that are non-supersymmetric but nevertheless have equal numbers of massless bosons and fermions $\left(N_{f}^{0}=N_{b}^{0}\right)$ have a one-loop cosmological constant that is exponentially suppressed. Ref. [15] argues that these cases are particularly interesting due to their enhanced stability properties, and form a better basis for doing phenomenology.

The philosophy in these cases is somewhat different: the general idea is that the exponential suppression appearing in the vacuum energy also appears in the scale setting the Higgs mass. Therefore the compactification volume (and consequently the SS supersymmetry breaking scale $1 / R$ ) needs to be only so large so as to be able to generate the necessary suppression, while it is possible to live with supersymmetry breaking that is much larger than the electroweak scale (the canonical situation with SS breaking). The issue for the 


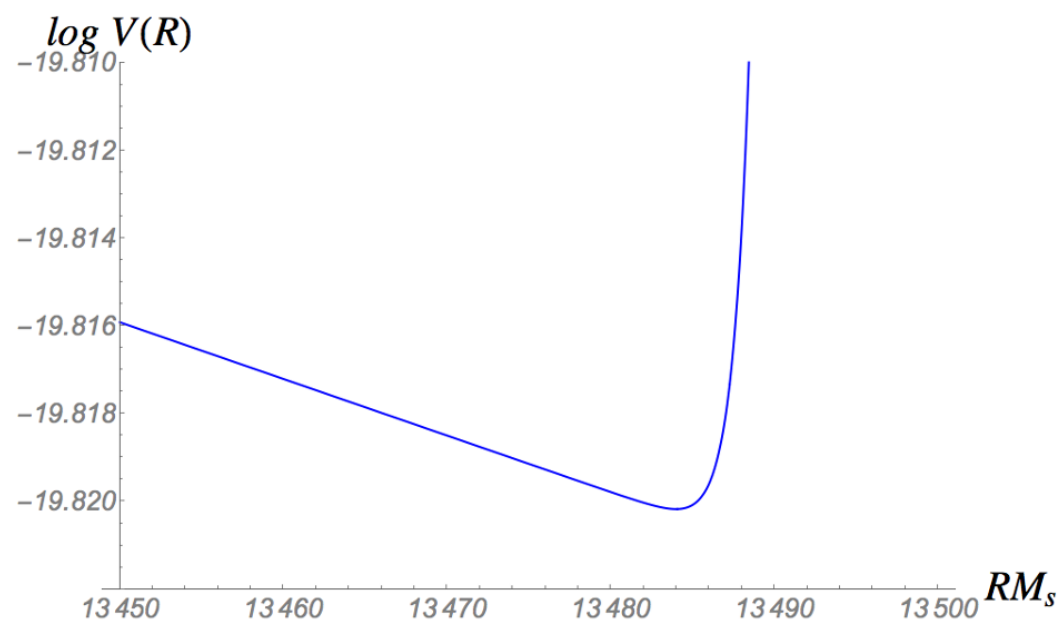

Figure 1. The 5D potential for $\alpha=\alpha_{D}=0.1, N_{f}^{0}-N_{b}^{0}=10, g_{s}=3 \times 10^{-2}, C=b=-13, N_{c}=6$. The approximation in eq. (2.14) gives the minimum at $R_{\min }=1.35 \times 10^{5} \ell_{s}$. As described in the text, the dynamical scale $\Lambda_{e} \approx 0.09 / R$ is significantly less than the KK scale at the minimum.

present discussion then is how to stabilise with exponentially small cosmological constant and reasonable coupling, but still with moderately large volume.

As already mentioned, the aspect of these theories that will be of particular relevance is that the only modes that make a non-vanishing contribution to the vacuum energy have string sized masses, and indeed the leading contribution to the Schwinger integral comes from a saddle-point at the UV end, $t \sim 1$, rather than from the entire integral, as is the case for a generic theory. Consequently the contribution to the cosmological constant resulting from the Scherk-Schwarz mechanism is blind to the IR physics occurring in for example the ISS mechanism, and the two contributions are physically separated. Indeed the former cannot easily be understood within an effective field theory. ${ }^{2}$

As already mentioned, to emphasise the distinction these theories will be said (using the terminology in its broad sense) to have a $U V$-Casimir energy. An additional advantage in the present context is of course that the volumes required are much smaller than the generic case, and hence the decompactification problem is less pronounced.

Assuming that the exponential suppression continues beyond one-loop, such cases have to be treated quite differently. In the present toy model, the ISS mechanism essentially governs the minimisation, and the issue is to ensure that the contribution to the cosmological constant from the initial SS mechanism is negligible. The 5D case is as follows.

First let us return to the constraints in eq. (2.1). The potential takes the form

$$
V=R^{-4}\left[\alpha^{2} \rho\left(N_{f}^{1}-N_{b}^{1}\right)\left(R M_{s}\right)^{2} e^{-4 \pi R M_{s}}+N_{c} \alpha_{D}^{2}\left(R \Lambda_{e}\right)^{2}\right],
$$

where $N_{f}^{1}-N_{b}^{1}$ counts the fermi-bose non-degeneracy at the first excited string level, $\alpha$ stands again for a generic Scherk-Schwarz phase, while $\rho \ll 1$ is now generically a one-loop

\footnotetext{
${ }^{2}$ Conceivably one could try to write down a supergravity theory truncated at the first string excitation level.
} 
suppression factor. As is evident from eq. (2.12) the SS term dies away rapidly at large radius. The minimum occurs shortly after the second term has its independent minimum at

$$
R_{\min } M_{s}=\frac{4 b}{C}
$$

so the string scale can be perhaps an order of magnitude higher than the KK scale. It is useful to define $\sigma \gtrsim 0$ as the final ratio of dynamical to KK scale, i.e. $R_{\min } \Lambda_{e}=e^{-\sigma}$, so that ultimately

$$
\sigma=\frac{8 \pi^{2}}{|b| g_{s}^{2}}-2 .
$$

As usual, $m_{D}$ must satisfy the constraints in eq. (2.1), so it lies below $\Lambda_{e}$ but is large enough that $\hat{m}_{D} \Lambda_{e}>\alpha^{2} / R^{2}$ :

$$
1 \gtrsim e^{-\frac{8 \pi^{2}}{|b| g_{s}^{2}}+2} \gg \alpha_{D}, \alpha^{2} / \alpha_{D}
$$

Thus for the mechanism to work when the Casimir energy is exponentially suppressed in the $5 \mathrm{D} \rightarrow 4 \mathrm{D}$ theory, the scale of supersymmetry breaking has to be at most a few orders of magnitude below the string scale with relatively large coupling, $8 \pi^{2} /|b| g_{s}^{2} \sim 2$. In addition $\alpha_{D} \sim \alpha$ are required to be small. Note that if these constraints are satisfied then the SS contribution to the cosmological constant is guaranteed to be negligible at the minimum, which was the point we wished to demonstrate here. The essential advantage of a UVCasimir energy in this case is that the (relatively) large volume stabilisation governed by non-perturbative long-range physics has not fed-back into it, so one has the sort of modularity normally associated with brane configurations.

As an example, taking $|C|=10,|b|=30, g_{s}=1 / \sqrt{2}$, one requires $\alpha, \alpha_{D} \lesssim R \Lambda_{e} \approx$ $1 / 25$. We will later see how to accommodate $\Lambda_{e}>M_{K K}$ in the $6 \mathrm{D} \rightarrow 4 \mathrm{D}$ version; this removes the upper bound in eq. (2.23) allowing maximal SS phases $\alpha_{D}, \alpha \sim 1$.

\section{String/supergravity embedding}

Let us now collect the components for a more complete implementation within a string compactification, focussing on a theory compactified to $\mathcal{N}=1$ in $6 \mathrm{D}$, and then further compactified on an orbifold of $\mathbb{T}_{2}$ down to $D=4$.

The discussion begins with a summary of the effective spontaneously broken supergravity theory and then compares the spectrum to that of the Scherk-Schwarzed string theory (using the framework of ref. [15]). The extension to 6D introduces modular symmetries that persist (as a congruence subgroup) in the Scherk-Schwarzed theory. It is shown that both the spectrum and the Casimir energy preserve these symmetries. Their great advantage is that they can be used to follow soft-terms in the spontaneously broken supergravity theory (taking over the role of the $R$-symmetry in global SQCD $[50,51]$ ).

This allows us to consider the theory as a whole, without having to separate supersymmetry breaking scales with artificially small SS twists as was done in the previous section. In fact we will ultimately find that the SS-induced soft-terms act to stabilise the minimum so that we do not have to rely on the one-loop metastability of ISS. 


\subsection{Spectrum and congruence subgroups in the effective supergravity theory}

First let us establish how the Scherk-Schwarz mechanism in a direct string implementation such as that in ref. [15] maps to the effective supergravity theory. As mentioned, the SS stage of compactification is on an orbifolded $\mathbb{T}_{2}$ torus, which in the absence of Wilson lines can be described generally by the metric

$$
G_{i j}=\frac{T_{2}}{U_{2}}\left(\begin{array}{cc}
1 & U_{1} \\
U_{1} & |U|^{2}
\end{array}\right) ; \quad G^{i j}=\frac{1}{T_{2} U_{2}}\left(\begin{array}{cc}
|U|^{2} & -U_{1} \\
-U_{1} & 1
\end{array}\right),
$$

where in order to conform with most of the phenomenology oriented SUGRA literature the convention is

$$
\begin{aligned}
& i U=U_{1}+i U_{2} \\
& i T=T_{1}+i T_{2} .
\end{aligned}
$$

For reference, untilted tori have $U_{1}=0, T_{2}=R_{1} R_{2}, U_{2}=R_{2} / R_{1}$ where $R_{i}$ is the radius along direction $i$, and it will be assumed throughout that $R_{2}>R_{1}$. The $U_{1}$ modulus encapsulates the tilt angle (i.e. $U_{1}=R_{2} \cos \theta / R_{1}, U_{2}=R_{2} \sin \theta / R_{1}$ ) and $T_{2}=R_{1} R_{2} \sin \theta$ is the volume. The nett effect on the spectrum of the Scherk-Schwarz action can be determined on the string theory side from the shift in the internal momenta, which can in turn be read off the partition function. The latter contains a factor

$$
\mathcal{Z}_{d, d}(G, B)=\frac{1}{|\eta(\tau)|^{2 d}} \sum_{\mathbf{n}, \mathbf{m}} q^{\alpha^{\prime} \mathbf{p}_{\mathbf{L}}^{2} / \mathbf{2}} \bar{q}^{\alpha^{\prime} \mathbf{p}_{\mathbf{R}}^{2} / \mathbf{2}}
$$

coming from the compactified toroidal directions. The momenta depend on the KK numbers $m_{1,2}$ and winding numbers $n^{1,2}$ of the $\mathbb{T}_{2}$ as

$$
\begin{aligned}
\mathbf{p}_{L}^{2} & =p_{L_{i}} G^{i j} p_{L_{j}} \\
p_{L_{j}} & =\frac{1}{\sqrt{2 \alpha^{\prime}}}\left(m_{j}+\left(B_{j k}+G_{j k}\right) n^{k}\right),
\end{aligned}
$$

and

$$
\begin{aligned}
\mathbf{p}_{R}^{2} & =p_{R_{i}} G^{i j} p_{R_{j}} \\
p_{R j} & =\frac{1}{\sqrt{2 \alpha^{\prime}}}\left(m_{j}+\left(B_{j k}-G_{j k}\right) n^{k}\right),
\end{aligned}
$$

where the notation throughout is as in ref. [15]. The Scherk-Schwarz action causes a discrete Lorentz rotation and boost involving the KK and winding numbers and the charge/momentum lattice, $\mathbf{Q}$, of the form

$$
\begin{aligned}
\mathbf{Q} & \rightarrow \mathbf{Q}-n^{i} \mathbf{e}_{i} \\
m_{i} & \rightarrow m_{i}+\mathbf{Q} \cdot \mathbf{e}_{i}-\frac{1}{2} \mathbf{e}_{i} \cdot \mathbf{e}_{j} n^{j} \\
B_{j k} \pm G_{j k} & \rightarrow B_{j k} \pm G_{j k}-\frac{1}{4} \mathbf{e}_{j} \cdot \mathbf{e}_{k},
\end{aligned}
$$


where $\mathbf{e}_{i=1,2}$ are vectors containing the Scherk-Schwarz action on the $R$-charges and possibly also gauge charges, and the dot product refers to the Lorentzian charge lattice. The vectors $\mathbf{e}_{i}$ contain the phases $\alpha_{F, B}$, although one should note they must leave the world sheet supercurrent and charge lattice invariant, and have to leave a consistent orbifold projection. It is for these reasons that $\alpha_{F, B}$ are constrained to be discrete.

Specialising to the maximal twist case, the spontaneous supersymmetry breaking arises from half integer values of the $\mathbf{Q} \cdot \mathbf{e}_{i}$ shift in the KK numbers. Consider the gravitinos; adding left and right moving contributions, the modes $m_{i}$ and $\mathbf{Q} \cdot \mathbf{e}_{i}$ marry with the modes $-m$ and $-\mathbf{Q} \cdot \mathbf{e}_{i}$, so that

$$
\begin{aligned}
\left(m_{3 / 2}^{\left(m_{1} m_{2}\right)}\right)^{2} & =\frac{1}{\alpha^{\prime}} \hat{m}_{i} G^{i j} \hat{m}_{j}=\frac{1}{4 \alpha^{\prime}} \sum_{i j} G^{i j} \\
& =\frac{1}{\alpha^{\prime}} \frac{1}{T_{2} U_{2}}\left|\left(m_{1}-\frac{1}{2}\right)-\left(m_{2}-\frac{1}{2}\right) i U\right|^{2} .
\end{aligned}
$$

Clearly supersymmetry is restored for all $U_{1}=\frac{2 \ell_{1}-1}{2 \ell_{2}-1}$ in the limit $U_{2} \rightarrow 0$ for integer $\ell_{1,2}$. This limit can be achieved by decompactifying with constant ratio of radii, with the tilt angle going to zero (slower than $1 / R_{1} R_{2}$ in order for $T_{2}$ to go to large volume). An identical mass-shift is induced in the gauginos. From this we can identify the effective KK scale near a supersymmetric point as $M_{K K}^{2}=U_{2} / T_{2}=1 / R_{1}^{2}$. (Where necessary factors of $\alpha^{\prime}$ are absorbed into the modulus $T$ to give it dimensions of length squared.)

Continuous Wilson lines shift the KK and winding numbers along with the internal charges in a similar fashion and these can be related to matter/Higgs fields: the shift induced by the pair of continuous real Wilson lines $\mathbf{A}_{1}, \mathbf{A}_{2}$, can be written

$$
\begin{aligned}
\mathbf{Q} & \rightarrow \mathbf{Q}+n^{i} \mathbf{A}_{i} \\
m_{i} & \rightarrow m_{i}-\mathbf{Q} \cdot \mathbf{A}_{i} \\
B_{j k} \pm G_{j k} & \rightarrow B_{j k} \pm G_{j k}-\frac{1}{4} \mathbf{A}_{j} \cdot \mathbf{A}_{k} .
\end{aligned}
$$

The real shift vectors $\mathbf{A}_{i}$ can be related to a pair of complex fields in the effective supergravity theory, denoted $\phi, \phi^{\prime}$. To get to this basis, first define complex Wilson lines,

$$
\mathbf{Z}=i U \mathbf{A}_{1}-\mathbf{A}_{2}
$$

and then

$$
\begin{aligned}
i \phi & =\frac{1}{2}\left(Z^{1}-i Z^{2}\right) \\
i \phi^{\prime} & =\frac{1}{2}\left(Z^{1}+i Z^{2}\right) .
\end{aligned}
$$

The upper indices refer to basis vectors for the charge lattice. Defining $2 P=\phi+\bar{\phi}^{\prime}$, useful combinations are (in our conventions)

$$
\begin{aligned}
P \bar{P} & =\sum_{a} \Im\left(Z^{a}\right)^{2}=\left(\mathbf{A}_{1} \cdot \mathbf{A}_{1}\right) \frac{U_{2}^{2}}{4} \\
T_{2} & =\sqrt{G}+P \bar{P} / U_{2} \\
U & =\frac{1}{G_{11}}\left(\sqrt{G}-i G_{12}\right),
\end{aligned}
$$


with the $T_{2}$ redefinition matching the shift in (3.8). Going from $\mathbf{Z}$ to $\phi, \phi^{\prime}$ amounts to a change of basis for $\mathbf{Q}$. For example the current superfield for a $\mathrm{U}(1)$ current (under which $\phi$ and $\phi^{\prime}$ must have opposite charges) is given by, $J=|\phi|^{2}-\left|\phi^{\prime}\right|^{2}=\frac{i}{2}\left(Z^{1} \bar{Z}^{2}-Z^{2} \bar{Z}^{1}\right)$, so its generator acts as $\mathrm{SO}(2)$ on the $Z^{a}$ indices. The Kähler potential depends on the volume $\sqrt{G}$ as

$$
K=-\log Y-\log 4\left(T_{2} U_{2}-P \bar{P}\right),
$$

where $2 P=\phi+\bar{\phi}^{\prime}$, and where the dilaton combination generally includes a term from the (heterotic) Green-Schwarz mechanism,

$$
Y=S+\bar{S}-\delta_{G S} \log 4\left(T_{2} U_{2}-P \bar{P}\right) .
$$

So far the picture is just that of the standard $\mathcal{N}=1$ theories, but now we deform the theory with a superpotential that successfully reproduces the SSSB observed in the string spectrum. As we saw on the string side in eq. (3.7), near $i U=1$ the lightest spin $3 / 2$ state is the zero-KK mode gravitino whose physical mass is

$$
m_{3 / 2}^{2}=\frac{1}{4} \frac{1}{S_{2} T_{2} U_{2}}|1-i U|^{2} .
$$

The relation between the Planck scale and string scale is

$$
M_{P}^{2}=g_{s}^{-2} \alpha^{\prime-1},
$$

which suggests that a superpotential in the spontaneously broken theory that produces the correct spectrum is

$$
W_{\mathrm{SS}}=\sqrt{2}(1-i U) .
$$

It can be verified that near $U_{1}=1$, the rest of the low-lying tree-level string spectrum is successfully generated by this supergravity theory. Explicitly, in the string spectrum the tree-level gaugino masses are degenerate with the gravitino: using standard notation, the supercovariant derivative is $D_{i} W=W_{i}+W K_{i}$, and the gauge kinetic function is $f_{\text {tree }}=S$, leading to

$$
m_{\lambda}=\left|\frac{m_{3 / 2}}{2} \operatorname{Re}\left(f_{\text {tree }}\right)^{-1} K^{\bar{i} j} \partial_{i} f_{\text {tree }} \frac{D_{\bar{j}} \bar{W}}{\bar{W}}\right|=m_{3 / 2} .
$$

At one-loop the masses would not be equal in either the field theory or the string theory due to gauge mediation effects, but we shall see below that the above relation does not suffer large volume corrections.

Continuing the comparison of the spectra, after spontaneous superymmetry breaking all the untwisted scalars in the NS-NS sector should remain massless at tree-level, while their fermion superpartners pick up a mass equal to that of the gravitino. The corresponding superfields, $\phi$ and $\phi^{\prime}$, achieve this by appearing to conspire in the Kähler potential as

$$
\begin{aligned}
K & \supset-\log \left(4 T_{2} U_{2}-\left|\phi+\bar{\phi}^{\prime}\right|^{2}\right) \\
& =-\log 4 T_{2} U_{2}+\frac{1}{4 T_{2} U_{2}}\left(|\phi|^{2}+\left|\phi^{\prime}\right|^{2}+\phi \phi^{\prime}+\bar{\phi} \bar{\phi}^{\prime}\right)+\ldots
\end{aligned}
$$


The tree-level fermion mass terms in the effective theory (which is a " $\mu$-term" if one is thinking of $\phi, \phi^{\prime}$ as Higgses), are then given by

$$
\mu_{\phi}=m_{3 / 2} Z_{\phi}^{-\frac{1}{2}} Z_{\phi^{\prime}}^{-\frac{1}{2}}\left(\frac{W_{i j}}{W}+K_{i j}-\Gamma_{i j}^{k} \frac{D_{k} W}{W}\right),
$$

where

$$
\begin{aligned}
\Gamma_{i j}^{k} & =K^{k \bar{k}} \partial_{\bar{k}} K_{i j} \\
Z_{\phi}^{-1}=Z_{\phi^{\prime}}^{-1} & =4 T_{2} U_{2}=1 / K_{\phi \phi^{\prime}} .
\end{aligned}
$$

Inserting the supersymmetry breaking superpotential in eq. (3.16) gives

$$
\mu_{\phi}=m_{3 / 2}\left(4 T_{2} U_{2} \frac{W_{i j}}{W}-\frac{\bar{W}}{W}\right)
$$

In the absence of any explicit $W_{i j}$ mass terms in the original superpotential, this automatically has the same magnitude as the gaugino and gravitino masses in accord with the Scherk-Schwarzed string theory spectrum. It is straightforward to show that $S, U, T, \phi$ and $\phi^{\prime}$ fit into a larger "no-scale" supergravity structure that leaves all the scalars massless at tree-level (modulo possible variations in the splittings of the matter fields that may arise if $\mathbf{e}$ is also embedded into the gauge groups: in the effective theory this would correspond to turning on scalar "Higgs" VEVs). The conspiring dimensionful terms correspond to mass-squareds and Dirac masses of magnitude $m_{3 / 2}$ for the canonically normalized states.

The original $\mathcal{N}=1$ theory has well-known modular symmetries: for completeness the standard $\mathrm{SL}(2, \mathbb{Z})_{T}$ and $\mathrm{SL}(2, \mathbb{Z})_{U}$ symmetries of the supersymmetric theory are included in the appendix. What remains of them after applying the Scherk-Schwarz mechanism? Due to the spontaneous nature of the breaking it is clear that the Kähler potential should still respect the full symmetry, as it indeed does, and that the new SSSB superpotential should be the only source of its breaking. To see its effect on the modular symmetries consider the spectrum: according to eq. (3.7) the zero-mode KK gravitino need not be the lightest state, depending on the value of $U_{1}$. If $U_{1}=\frac{2 \ell_{1}-1}{2 \ell_{2}-1}$ then the lightest gravitino is instead the $\ell_{1}, \ell_{2} \mathrm{KK}$ mode for all $U_{2} \lesssim 1 /\left(2 \ell_{2}+1\right)$, and the superpotential in the effective theory would actually be $W_{\mathrm{SS}}=\sqrt{2}\left(\left(2 \ell_{1}-1\right)-\left(2 \ell_{2}-1\right) i U\right)$ near this point. The fact that one has to specify which mode plays the role of the gravitino in the effective theory is of course just a symptom of the deficiency of the $4 \mathrm{D}$ supergravity approximation, which cannot describe the supersymmetry breaking over the whole $U$ moduli-space. Indeed the explicit breaking of modular symmetry in the superpotential just amounts to a choice of gauge: because of the original discrete symmetry, there are infinitely many equivalent spontaneously broken theories that one could write down for the effective supergravity theory related by a subgroup of the $\mathrm{SL}(2, \mathbb{Z})_{U}$ transformations. This is evident from the fact that under transformations of the form

$\frac{1}{4} \frac{|1-i U|^{2}}{S_{2} T_{2} U_{2}} \equiv \frac{1}{4} \frac{|(d-b)-(a-c) i U|^{2}}{S_{2} T_{2} U_{2}} ; a, b, c, d \in \mathbb{Z}, a d-b c=1, a-c=b-d=1 \bmod (1)$, 


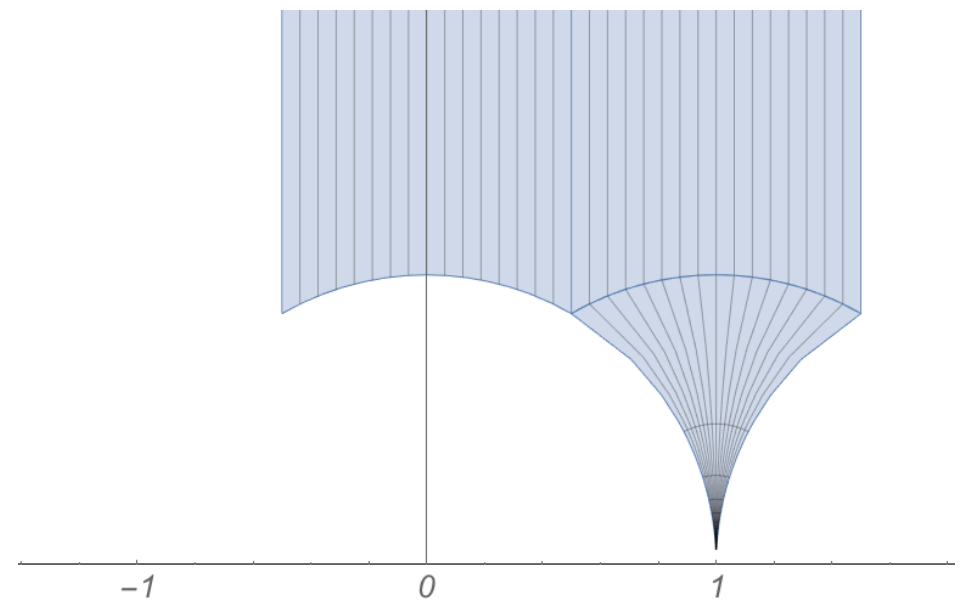

Figure 2. The fundamental $U$-modulus domain for a maximally twisted Scherk-Schwarz theory has a supersymmetric cusp at $i U=1$.

the gravitino spectrum is invariant. In fact the entire theory is invariant only under the smaller congruence subgroup defined by $a, d=1 \bmod (1)$ and $b, c=0 \bmod (1)$, similar to ref. [38], which will be referred to as $\Gamma_{1}(2)$. Under such transformations, any $U$ in a maximally twisted Scherk-Schwarz theory can be mapped to the fundamental domain shown in figure 2. In addition to the cusp at infinity, there is a single representative supersymmetric cusp at $i U=1$. For non-maximal Scherk-Schwarz twists, the fundamental domain will contain more cusps, and there will be several genuinely distinct supersymmetric vacua (consult ref. [38] for details). Naturally the Casimir energy, when we come to calculate it, must respect this symmetry.

We will also need an understanding of the one-loop gauge thresholds. Their volume dependence (neglecting the effects of extra charged massless states) can be written [38]

$$
\Delta=-C \log \left(T_{2} U_{2}|\eta(i T)|^{4}|\eta(i U)|^{4}\right)+(C-b) \log \left(T_{2} U_{2}\left|\vartheta_{4}(i T)\right|^{4}\left|\vartheta_{2}(i U)\right|^{4}\right),
$$

where $b=16 \pi^{2} \beta$ is the beta function coefficients for the entire massless theory, $C=$ $16 \pi^{2} \beta_{\mathcal{N}=2}$ is the $N=2$ coefficient, and $\eta$ are the usual Dedekind eta functions. The modular functions in this expression are also invariant under $\Gamma_{1}(2)$ transformations; denoting $\mathrm{SL}(2, \mathbb{Z})_{U}$ operations by $S_{U} \equiv i U \rightarrow-1 / i U$ and $T_{U} \equiv i U \rightarrow i U+1$, we have

$$
\begin{gathered}
T_{U}: U_{2}\left|\vartheta_{2}(i U)\right|^{4} \longrightarrow U_{2}\left|\vartheta_{2}(i U)\right|^{4} \\
S_{U}: U_{2}\left|\vartheta_{2,4}(i U)\right|^{4} \longrightarrow U_{2}\left|\vartheta_{4,2}(i U)\right|^{4} .
\end{gathered}
$$

Therefore $\Delta$ is invariant under any number of $T_{U}$ moves, but only an even number of $S_{U}$ moves, in accord with the congruence condition.

Following now the standard route (see for example refs. [52-55]) this allows us to identify the holomorphic gauge kinetic function of the SQCD as (taking a Kac-Moody level $k=1$ for the gauge group),

$$
f=S-\frac{C}{8 \pi^{2}} \log \eta(i T)^{2} \eta(i U)^{2}+\frac{C-b}{8 \pi^{2}} \log \left(\vartheta_{4}(i T)^{2} \vartheta_{2}(i U)^{2}\right),
$$


with the gauge coupling being given by

$$
\frac{2}{g^{2}}=Y=2 \Re(f)-\frac{b}{8 \pi^{2}} \log \left(\mu^{2}\right)-\left(\frac{b}{8 \pi^{2}}+\delta_{G S}\right) \log \left(4 T_{2} U_{2}\right) .
$$

Note that due to the additional universal terms it is the $\mathcal{N}=1$ beta function appearing here (i.e. $b=-3 N+F$ in $\mathrm{SU}(N)$ gauge theories with $\mathcal{N}=1 \mathrm{SQCD}$ and $F$ flavours), and not $C$.

The holomorphic dynamical scale $\Lambda_{\text {hol }}$ can be defined as

$$
\Lambda_{\text {hol }}=\exp \left(-\frac{8 \pi^{2}}{|b|} f\right)
$$

and the modular weight of $\Lambda_{\text {hol }}$ is given by

$$
n_{\Lambda}=8 \pi^{2} \frac{b / 8 \pi^{2}+\delta_{G S}}{|b|} .
$$

The gauge coupling can then be written more succinctly as

$$
\frac{1}{g^{2}(\mu)}=-\frac{b}{8 \pi^{2}} \log \left[\frac{\mu}{\left|\Lambda_{\text {hol }}\right|\left(4 T_{2} U_{2}\right)^{n_{\Lambda} / 2}}\right]
$$

It will often be useful to leave $n_{\Lambda}$ implicit, as it is essentially just whatever combination of terms appears in eq. (3.27). However it can be calculated directly [53]; specialising to $\mathrm{SU}(N)$ gauge theories with $\mathcal{N}=1 \mathrm{SQCD}$ and $F$ flavours of quark and anti-quark, it is

$$
|b| n_{\Lambda}=2 F n_{Q}+F-N .
$$

We will see that this equation provides an important consistency condition for the implementation of the ISS mechanism, because it can be derived independently from the matching conditions for the Seiberg duals. Note that it will be assumed for simplicity throughout that the $\mathrm{SL}(2, \mathbb{Z})_{U}$ and $\mathrm{SL}(2, \mathbb{Z})_{T}$ weights are degenerate for every field.

To complete this part of the discussion, one can obtain an asymptotic approximation for the gauge threshold correction at large volume and in the supersymmetric limit around the representative cusp at $i U=1$ (which obviously breaks the modular symmetry). In the vicinity of the cusp, since $\lim _{i U \rightarrow 1} \eta(i U)=0$, it is often convenient to use $\operatorname{SL}(2, \mathbb{Z})_{U}$ modular redefinitions to the cusp at infinity, that is $i \tilde{U}=-1 /(i U-1) \approx i / U_{2}$, with $i \tilde{U} \rightarrow i \infty$ in the supersymmetric limit: the standard expansion $\vartheta_{4}(i \tilde{U}) \rightarrow 1-2 e^{-\pi \tilde{U}}+\ldots$ then gives,

$$
\begin{aligned}
\Delta & =-C \log \left(4 T_{2} \tilde{U}_{2}|\eta(i T)|^{4}|\eta(i \tilde{U})|^{4}\right)+(C-b) \log \left(4 T_{2} \tilde{U}_{2}\left|\vartheta_{4}(i T)\right|^{4}\left|\vartheta_{4}(i \tilde{U})\right|^{4}\right) \\
& =\frac{\pi}{3} C\left(T_{2}+\tilde{U}_{2}\right)-b \log \left(4 T_{2} \tilde{U}_{2}\right)+\mathcal{O}\left(e^{-\pi \tilde{U}_{2}}, e^{-\pi T_{2}}\right) .
\end{aligned}
$$

As in the $5 \mathrm{D}$ case, the second term subtracts from $16 \pi^{2} / g(\mu)^{2}$ the logarithmic running between the lightest KK-mode $M_{K K}=1 / \sqrt{4 T_{2} \tilde{U}_{2}}$ and the string scale, whilst the first term replaces it with a power-law threshold. Under our assumption that $C / b>0$, it is 
clear that one is prevented from going continuously to the boundary of moduli-space by the appearance of strong coupling in the QCD theory where $\frac{\pi}{3} C\left(T_{2}+\tilde{U}_{2}\right) \sim 16 \pi^{2}$, and this is precisely the region in which the minimum is expected to appear.

Returning to the appearance of the large volume dependence in the one-loop gaugino mass, retaining only the pieces $f \approx S+\frac{C}{8 \pi^{2}} \frac{\pi}{6}(T+\tilde{U})$, eq. (3.17) and a little work shows that the relation $m_{\lambda}=m_{3 / 2}$ holds at one-loop up to logarithmic corrections, as promised.

\subsection{Calculation of Casimir energy}

Next let us determine the cosmological constant for the general $6 D \rightarrow 4 D$ case, essentially repeating the computation of ref. [15] in the full string theory, but now retaining the full $T, U$ dependence. In particular it will be possible to check that the result respects the $\Gamma_{1}(2)$ symmetry of the congruence subgroup described above.

The required expression is

$$
\Lambda^{(4)}(T, U)=-\frac{1}{2} \int_{\mathcal{F}} \frac{d^{2} \tau}{\tau_{2}^{2}} \mathcal{Z}(\tau) .
$$

Using the result in eq. (3.6), the partition function can be approximated at large volume $\left(T_{2} \gg 1\right)$ by neglecting the winding modes and Poisson resumming the KK modes of eq. (3.3), giving

$$
\mathcal{Z}_{\mathbf{0}, \ell}=\frac{\mathcal{M}^{2}}{\tau_{2}|\eta|^{4}} \sqrt{\operatorname{det} G} e^{-\frac{\pi}{\tau_{2}} \ell^{i} G_{i j} \ell^{j}}
$$

The main simplifying approximation we are making is to neglect the non-zero winding mode contributions (i.e. $\mathcal{Z}_{n \neq \mathbf{0}, \ell}$ ) because they are suppressed by exponential factors when the volume is large. Indeed the largest possible terms with non-zero winding would come from otherwise massless modes with $n_{i}=1$, and would be proportional to $\sim e^{-\pi T_{2}} / \pi T_{2}$. This should be compared to the leading $n_{i}=0$ contributions which as in ref. [15] have a milder exponential suppression factor of $e^{-2 \pi \sqrt{T_{2}}}$. The $n_{i}=0, \sum_{i} \ell_{i}=$ even contributions remain supersymmetric regardless of the presence or otherwise of Wilson lines (assuming the latter do not themselves break supersymmetry), and therefore we need only consider $\ell_{1}+\ell_{2}=o d d$. In addition one can ignore the various twisted sectors of the orbifold which, being independent of the moduli, are supersymmetric and cannot contribute to $\Lambda$. As a further approximation one may at large volume neglect the non-level matched terms which allows one to express the result entirely in terms of physical states; the leading contributions being neglected in this latter approximation are from the proto-graviton state described in ref. [15], and are of order $\sim T_{2} e^{-\pi T_{2}}$. In making these approximations one obviously at this point has to abandon the full $\operatorname{SL}(2, \mathbb{Z})_{T}$ modular structure of $\Lambda(T, U)$, but the $\Gamma_{1}(2)$ $U$-symmetry should remain. We are henceforth obliged to always work at large $T_{2}$ (which just affirms the preamble concerning the importance of interpolation).

The result is an expression for the partition function of the form

$$
\mathcal{Z}(\tau) \approx \frac{\mathcal{M}^{2}}{\tau_{2}|\eta|^{4}} \frac{1}{\eta^{8} \bar{\eta}^{20}} \sum_{\boldsymbol{\ell}} \mathcal{Z}_{\mathbf{0}, \ell} \sum_{\alpha, \beta} e^{2 \pi i \sum_{i} \ell_{i}[\mathbf{e} \cdot \mathbf{Q}]} \mathcal{Z}_{\text {internal }}\left[\begin{array}{c}
\alpha \\
\beta
\end{array}\right],
$$


where $\alpha, \beta$ label the sectors along the two cycles of the torus. Written as a sum over the physical states this reduces to

$$
\mathcal{Z}(\tau) \equiv \frac{T_{2}}{\tau_{2}^{2}} \sum_{\substack{\ell=\text { odd } \\ \text { level }=k}}\left(N_{b}^{(k)}-N_{f}^{(k)}\right) e^{-\frac{\pi}{\tau_{2}} \ell^{i} G_{i j} \ell^{j}} e^{-\pi \tau_{2} \alpha^{\prime} m_{k}^{2}},
$$

where $\left(N_{b}^{(k)}-N_{f}^{(k)}\right)$ is the Bose-Fermi non-degeneracy of the states unshifted by the ScherkSchwarz mechanism at level $k$. Inserting into eq. (3.33) this gives a leading contribution to the cosmological constant of

$$
\Lambda(T, U)=\frac{2}{\pi^{3}} \frac{1}{T_{2}^{2}}\left(N_{f}^{0}-N_{b}^{0}\right)\left[\frac{1}{2} \sum_{\ell_{1}+\ell_{2}=\text { odd }} \frac{U_{2}^{3}}{\left|\ell_{1}+i U \ell_{2}\right|^{6}}\right] .
$$

The sum in the square brackets, which will be referred to as $E_{3}(i U)$, is an Eisenstein series, restricted to odd $\ell_{1}+\ell_{2}=1 \bmod (1)$, instead of the canonical $\left(\ell_{1}, \ell_{2}\right) \neq(0,0)$. One can easily see that it indeed respects the congruence subgroup obeyed by the spectrum, and also that it has zeros at the supersymmetric points: indeed since $U_{1}=\left(2 \ell_{1}+1\right) /\left(2 \ell_{2}+1\right)$ implies $\left|m_{1}+U_{1} m_{2}\right| \geq 1 /\left(2 \ell_{2}+1\right) \forall \ell_{1}+\ell_{2} \bmod (1)=1$, one may smoothly take the $U_{2} \rightarrow 0$ limit of the sum for precisely these values. In accord with the modular transformation above, there is an infinite number of such "trivial zeros", at all odd integer values of $U_{1}$ as well as fractions with odd numerator and denominator, with the general structure as one approaches the $U_{2}=0$ line becoming extremely intricate to reflect its modular symmetry, as shown in figure 3 . (It is not clear if anything interesting happens at irrational values of $U_{1}$.)

For use in the minimisation let us focus on the Casimir energy around the representative supersymmetric cusp at $i U=1$. The potential near $i U=1$ is shown in figure 3. Clearly the minimisation will take place near $|U|=1$ and the phase of $U$ will be the dynamically important variable. The potential along the unit circle is also shown, along with the following approximation which can be evaluated in closed form:

$$
E_{3}(i U) \approx 2 \sum_{k} \frac{U_{2}^{3}}{|2 k+i U|^{6}} \rightarrow \frac{\pi^{6} U_{2}^{3}}{240}
$$

The $N_{f}^{0}=N_{b}^{0}$ case is instead dominated by the leading saddle point. According to eqs. (3.33) and (3.36) we find

$$
\begin{aligned}
\Lambda(T, U) & =\frac{T_{2}}{2}\left(N_{f}^{1}-N_{b}^{1}\right) \sum_{\ell_{1}+\ell_{2}=\text { odd }}\left(\ell^{i} G_{i j} \ell^{j}\right)^{-7 / 4} e^{-2 \pi \sqrt{\ell^{i} G_{i j} \ell^{j}}} \\
& =\frac{\left(N_{f}^{1}-N_{b}^{1}\right)}{2} T_{2}^{-3 / 4} U_{2}^{7 / 4} \sum_{\ell_{1}+\ell_{2}=\text { odd }} \frac{e^{-2 \pi \sqrt{T_{2} / U_{2}}\left|\ell_{1}+i U \ell_{2}\right|}}{\left|\ell_{1}+i U \ell_{2}\right|^{7 / 2}} .
\end{aligned}
$$

Expanding about $i U \approx 1$ the following approximation will be useful:

$$
\Lambda(T, U)=2\left(N_{f}^{1}-N_{b}^{1}\right) T_{2}^{-3 / 4} U_{2}^{7 / 4} e^{-2 \pi \sqrt{T_{2} / U_{2}}}(1+\mathcal{O}(i U-1))
$$




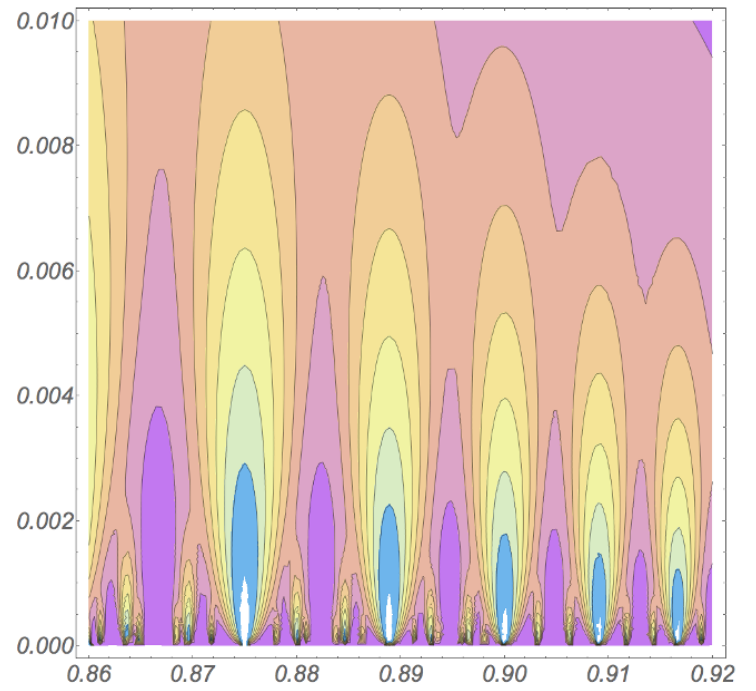

(a)

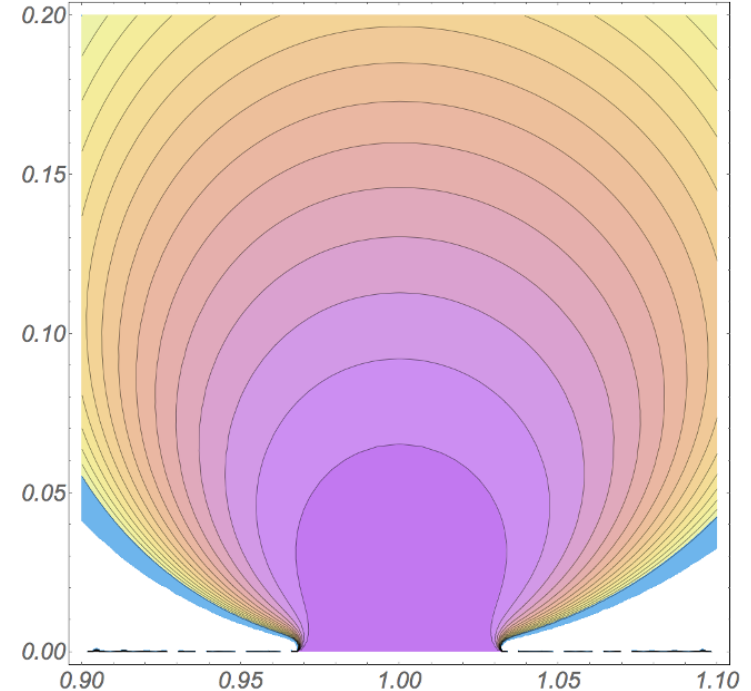

(b)

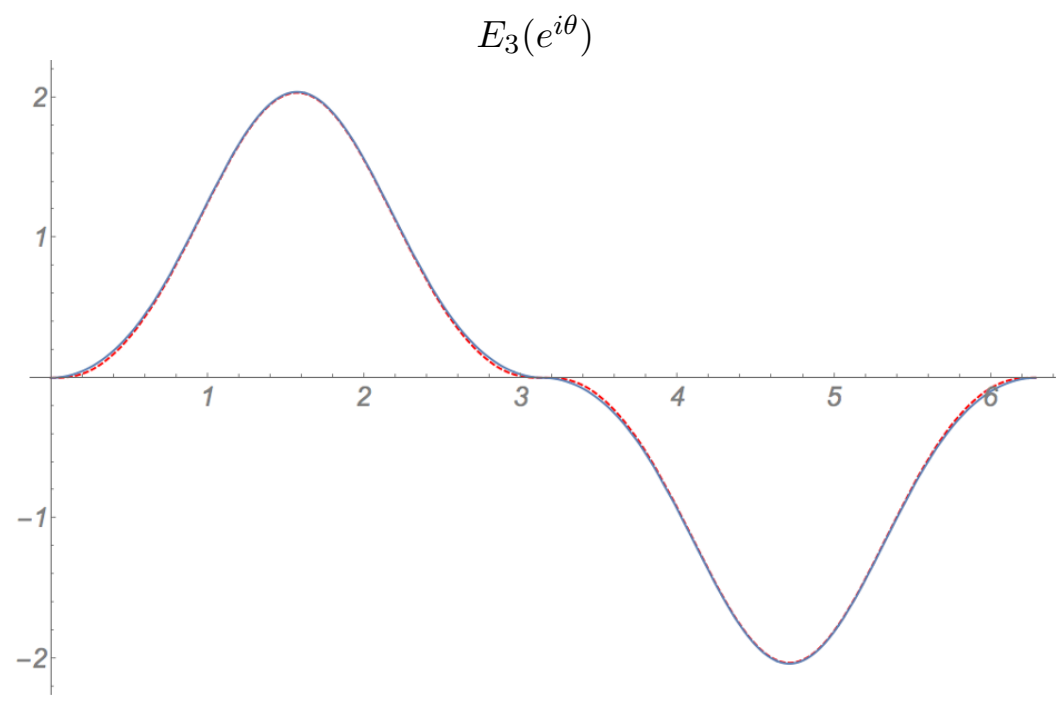

(c)

Figure 3. The Casimir energy $E_{3}(i U)$. In a) we see the self-similarity near the critical line, with the bottom of each valley corresponding to $U_{1}=\left(2 \ell_{1}+1\right) /\left(2 \ell_{2}+1\right)$ for integer $\ell_{1}, \ell_{2}$, and a different gravitino. Figure b) shows the vacuum energy around $i U=1$ as a function of $\rho, \theta$ where $i U=\rho e^{i \theta}$, and figure c) shows it along the unit circle $i U=e^{i \theta}$. The dashed line is the approximation $E_{3}(i U) \approx 2 \sum_{k} \frac{U_{2}^{3}}{|2 k+i U|^{6}}$. 


\subsection{The congruence subgroup method for mapping soft-terms between Seiberg duals}

Next we determine how the ISS mechanism is governed by the congruence subgroup. This subsection contains two new results. First it is shown that the string relation between the modular weights in eq. (3.31) can be derived as the unique solution to modular invariance in a pair of Seiberg duals, and secondly it is shown that the congruence subgroup provides a useful means of tracking soft-terms, including the effect of gravity mediation. It is also shown that the ISS mechanism still operates, with all masses, dynamical scales and so forth being replaced by the corresponding physical and hence modular invariant quantities. The issue of how KK modes enter into the ISS mechanism will be addressed in the following subsection.

Recall that in the ISS mechanism, the original electric theory has a Dirac mass superpotential,

$$
W_{\mathrm{el}}=m_{D} Q \tilde{Q}
$$

while the magnetic dual has a superpotential

$$
W_{\mathrm{mag}}=\frac{[Q \tilde{Q}] q \tilde{q}}{\hat{\Lambda}}+m_{D}[Q \tilde{Q}]
$$

The inverse coupling $\hat{\Lambda}$ in the superpotential is expected to be of order the strong coupling scale of the theory. One can determine its modular weight from the requirement that $W_{\text {mag }}$ has weight -1 , as does the dynamically induced superpotential for the SQCD theory,

$$
W_{\mathrm{dyn}}=-\tilde{N}\left(\frac{\operatorname{det}_{F}[Q \tilde{Q}]}{\hat{\Lambda}^{3 N-F}}\right)^{1 / \tilde{N}}
$$

This yields the modular weights of $Q$ and $q$ in terms of the weight of $\hat{\Lambda}$ :

$$
\begin{aligned}
n_{q} & =n_{\hat{\Lambda}} \frac{2 F-3 N}{2 F}-\frac{N}{2 F} \\
n_{Q} & =n_{\hat{\Lambda}} \frac{3 N-F}{2 F}-\frac{F-N}{2 F} .
\end{aligned}
$$

A nontrivial consistency check is that these expressions are in accord with the string relations in eq. (3.31) in both the electric and magnetic phases. They are also in accord with the well known matching relation,

$$
\Lambda_{\mathrm{hol}}^{-b} \tilde{\Lambda}_{\mathrm{hol}}^{-\tilde{b}} \sim \hat{\Lambda}^{-F}
$$

as well as the matching of baryons,

$$
\left(\frac{Q}{\Lambda_{\mathrm{hol}}}\right)^{N} \sim\left(\frac{q}{\tilde{\Lambda}_{\mathrm{hol}}}\right)^{\tilde{N}},
$$


provided that $n_{\hat{\Lambda}_{\text {hol }}}=n_{\Lambda_{\text {hol }}}=n_{\tilde{\Lambda}_{\text {hol }}}$, where $\Lambda_{\text {hol }}$ and $\tilde{\Lambda}_{\text {hol }}$ are the electric and magnetic QCD scales respectively. Their weights will be referred to collectively as $n_{\Lambda}$. The weight of the Dirac mass is then constrained to be

$$
n_{m_{D}}=-n_{\Lambda} \frac{3 N-F}{F}-\frac{N}{F} .
$$

As the three scales have the same modular weights, there can be no relative factors of $T_{2}$ or $U_{2}$ between them, and it is natural to assume $\hat{\Lambda} \sim \tilde{\Lambda}_{\text {hol }} \sim \Lambda_{\text {hol }}$. For example, if the fields $Q$ and $\tilde{Q}$ are incorporated into the "no-scale" structure such that they have weight $n_{Q}=-1$, then the corresponding modular weights of $\Lambda_{\text {hol }}$ and $m_{D}$ are $n_{\Lambda}=-(N+F) /(3 N-F)$ and $n_{m_{D}}=1$ respectively.

Finally the holomorphic magnetic meson is defined as

$$
\Phi=\frac{[Q \tilde{Q}]}{\Lambda_{\mathrm{hol}}} .
$$

It has weight

$$
n_{\Phi}=n_{\Lambda}\left(\frac{3 N-2 F}{F}\right)-\frac{F-N}{F} .
$$

Note that the dependence on $n_{\Lambda}$ in eq. (3.44) is proportional to the beta function in the respective theory, and at fixed points the modular weights of fields are proportional to their anomaly-free $R$-charges in the global theory. Thus when $F \approx 3 N / 2$ and the magnetic theory is weakly coupled, $n_{q} \approx n_{\Phi} \approx-1 / 3$, which can be interpreted as the appropriate modular weight for them to become free fields at a Gaussian fixed point. Likewise the weakly coupled electric theory, when $F \approx 3 N$, has $n_{Q} \approx-1 / 3$. In addition note that a non-zero value for $m_{D}$ breaks both the anomaly-free $R$-symmetry of the global theory, and the modular symmetry.

How are these objects related to their physical counterparts? The physical mass of the quarks is determined by the Kähler piece, $K \supset\left(|Q|^{2}+|\tilde{Q}|^{2}\right)\left(4 T_{2} U_{2}\right)^{n_{Q}}$, so the canonically normalized quark is $\hat{Q}=Q\left(4 T_{2} U_{2}\right)^{n_{Q} / 2}$, while the physical mass is $\hat{m}_{D}=$ $e^{K / 2} W_{Q \tilde{Q}}\left(4 T_{2} U_{2}\right)^{-n_{Q}}=m_{D}\left(4 T_{2} U_{2}\right)^{-\left(n_{Q}+1 / 2\right)}$. Both are modular invariant as they should be. We must also be careful to distinguish the holomophic scale $\Lambda_{\text {hol }}$ from the physical dynamical scale of the theory $\Lambda_{e}$. The two are related through the gauge thresholds according to eq. (3.30), which yields

$$
\Lambda_{e}=\left|\Lambda_{\mathrm{hol}}\right|\left(4 T_{2} U_{2}\right)^{\frac{n_{\Lambda}}{2}} .
$$

Thus the physical scale $\Lambda_{e}$ can be different from the holomorphic one, but note that in principle they can be similar in size, even at large volume: restoring the explicit radii and tilt dependence, $U_{1} \approx 1 \Longrightarrow R_{1}=R_{2} \cos \theta$, and hence $T_{2} U_{2} \approx R_{2}^{2}-R_{1}^{2}$. One may always choose $R_{2}^{2} \approx R_{1}^{2}+c^{2}$ where $c$ is an $\mathcal{O}(1)$ constant, so that $T_{2} U_{2} \approx c^{2}$. In this limit the tilt angle is very small, $\sin \theta \approx c / R_{2}$. This will turn out to be the dynamically relevant limit for the minimisation.

In the large $T_{2}$ and $\tilde{U}_{2}$ limit, eq. (3.32) gives,

$$
\frac{16 \pi^{2}}{g^{2}(\mu)}=\frac{16 \pi^{2}}{g_{s}^{2}}+\frac{\pi}{3} C\left(T_{2}+\tilde{U}_{2}\right)-b \ln \left(\mu^{2} 4 T_{2} \tilde{U}_{2}\right)
$$


and hence an approximation for $\Lambda_{e}$,

$$
4 T_{2} \tilde{U}_{2} \Lambda_{e}^{2}=e^{-\frac{16 \pi^{2}}{g_{s}^{2}|b|}+\frac{C}{b} \frac{\pi}{3}\left(T_{2}+\tilde{U}_{2}\right)} .
$$

Since this approximation is valid only in the specific $i U \rightarrow 1$ limit, it is unsurprisingly not modular invariant. Indeed the physical KK scale is a non-modular invariant quantity, and is given by the splitting in the spectrum, $M_{K K}=1 / \sqrt{4 T_{2} \tilde{U}_{2}}$. As $T_{2} \tilde{U}_{2} \equiv R_{1}^{2}$ it is, unlike $T_{2} U_{2}$, inevitably large.

The story for the physical magnetic meson is less clear-cut because it is not possible to determine the normalization precisely. However, given the modular weight of $\Phi$, it is reasonable to adopt an invariant Kähler potential of (up to irrelevant factors)

$$
K \supset|\Phi|^{2}\left(4 T_{2} U_{2}\right)^{n_{\Phi}}+\ldots
$$

Thus we work with a normalized field $\hat{\Phi}=\Phi / \gamma$, where $\gamma \equiv\left(4 T_{2} U_{2}\right)^{-n_{\Phi} / 2}$. The canonically normalized field is the modular invariant combination, $\hat{\Phi}=\hat{Q} \hat{\tilde{Q}} / \Lambda_{e}$. In the free-magnetic window where the ISS mechanism operates,

$$
-\frac{1}{3} \leq n_{\Phi} \lesssim 1
$$

with the lower limit corresponding to $2 F=3 N$.

The aspect of SQCD that we wish to address with this technology is the behaviour of the soft supersymmetry breaking terms that are induced in the original theory by the SS mechanism. In global theories such terms can be followed, even through regions of strong coupling, using various tools, most notably the $R$-current superfield, as described in refs. $[50,51]$. For example, properly normalized gaugino masses in the original SQCD electric theory are mapped to the magnetic dual as

$$
m_{g}^{(m a g)}=\frac{2 F-3 N}{3 N-F} m_{g}^{(e l)} .
$$

There is a similar (and related) mapping of mass-squared operators for the squarks and smesons, which in the global theory looks like

$$
|\hat{Q}|^{2}+|\hat{\tilde{Q}}|^{2} \rightarrow\left(\frac{2 F-3 N}{3 N-F}\right)\left[|\hat{q}|^{2}+|\hat{\tilde{q}}|^{2}-|\hat{\Phi}|^{2}\right] .
$$

These mappings in softly broken global SQCD theories parametrically suppress the supersymmetry breaking when the theory is just inside the free magnetic window $2 F \lesssim 3 N$.

In a similar fashion, modular symmetry can track the soft-terms in the effective supergravity theory. Due to its holomorphic nature the gaugino mass mapping is unchanged. But the mapping for the mass-squareds is different. Indeed a little work shows that a generic canonically normalized matter field $\hat{\varphi}$ has soft mass-squared terms

$$
m_{\hat{\varphi}}^{2}=m_{3 / 2}^{2}\left(1+2 n_{\varphi}\right)+\ldots
$$

where the dots indicate loop corrections. Numerical factors in the normalisation obviously cancel out in the physical mass-squared which depends only on the modular weights (which 
is why it was safe to ignore them). In the SQCD supergravity theories, this gives the following mapping of soft-terms:

$$
\begin{aligned}
& m_{\hat{Q}}^{2}=m_{3 / 2}^{2}\left[\frac{(3 N-F)}{F} n_{\Lambda}+\frac{N}{F}\right] \\
& m_{\hat{q}}^{2}=m_{3 / 2}^{2}\left[\frac{(2 F-3 N)}{F} n_{\Lambda}+\frac{F-N}{F}\right] \\
& m_{\hat{\Phi}}^{2}=m_{3 / 2}^{2}\left[-\frac{2(2 F-3 N)}{F} n_{\Lambda}+\frac{2 N-F}{F}\right] .
\end{aligned}
$$

One concludes that the relation in eq. (3.56) is not valid in the local theory, but that it would hold if one were to add a universal $-\frac{1}{3} m_{3 / 2}^{2}$ constant to all the soft-terms. Combined with the " 1 " in eq. (3.57), this extra $\frac{2}{3} m_{3 / 2}^{2}$ contribution is precisely the gravity mediated piece that is removed by the conformal compensator technique of ref. [50]. Here it is a real physical effect, and leads to an interesting sum-rule,

$$
2 m_{\hat{q}}^{2}+m_{\hat{\Phi}}^{2}=m_{3 / 2}^{2} .
$$

The right-hand side of this equation - which would be zero in a global theory - arises entirely from gravity mediation. This sum-rule implies that, in contrast to the global theory, there is now no choice of parameters that restores supersymmetry in the magnetic theory.

Eq. (3.57) cannot be the whole story for the scalar masses: for example no-scale models have massless scalars that have $n_{Q}=-1$. The additional contribution is of course from the cross-term in $K \supset\left|Q+\tilde{Q}^{\dagger}\right|^{2}\left(4 T_{2} U_{2}\right)^{n_{Q}}$. For models of this form one finds a dimensionful mass-squared operator in the potential for the canonically normalized fields of the form

$$
V_{\mathrm{el}} \supset m_{3 / 2}^{2}\left(1+n_{Q}\right)\left|\hat{Q}+\hat{\tilde{Q}}^{\dagger}\right|^{2}+\ldots
$$

The global flavour symmetry is explictly broken as

$$
\mathrm{SU}(F)_{L} \times \mathrm{SU}(F)_{R} \times \mathrm{U}(1)_{B} \times \mathrm{U}(1)_{R} \rightarrow \mathrm{SU}(F)_{V} \times \mathrm{U}(1)_{B},
$$

by the cross term. All $D$-flat scalar degrees of freedom remain massless when supersymmetry is spontaneously broken, and imposing these constraints on the magnetic description (as well as the flavour symmetry breaking pattern), fixes the magnetic Kähler potential to be

$$
K_{\text {mag }} \supset\left|q^{\dagger}+\tilde{q}\right|^{2}\left(4 T_{2} U_{2}\right)^{n_{q}}+\left|\Phi^{\dagger}+\Phi\right|^{2}\left(4 T_{2} U_{2}\right)^{n_{\Phi}},
$$

with the anti-hermitian part of $\Phi$ remaining massless, but the hermitian and trace parts picking up a mass of order $m_{3 / 2}^{2}$. This gives soft-terms of the form

$$
V_{\text {mag }} \supset m_{3 / 2}^{2}\left(1+n_{q}\right)\left|\hat{q}^{\dagger}+\hat{\tilde{q}}\right|^{2}+m_{3 / 2}^{2}\left(1+n_{\Phi}\right)\left|\hat{\Phi}^{\dagger}+\hat{\Phi}\right|^{2},
$$

up to normalisation factors that are irrelevant to the physical masses.

Finally with the above information to hand it is possible to check that the relevant physical processes respect the modular symmetry. For example a superpotential can be written for the canonically normalized fields of the effective global theory:

$$
\hat{W}(\hat{\Phi}, \hat{q}, \hat{\tilde{q}})=W e^{-\langle K\rangle / 2}=h \hat{\Phi} \hat{q} \hat{\tilde{q}}-\hat{m}_{D} \Lambda_{e} \hat{\Phi},
$$


where $h=\Lambda_{\text {hol }} / \hat{\Lambda}$ is a modular invariant coupling. The conclusion is that the typical induced physical mass scale in the ISS minimum is $\hat{\mu}=\sqrt{\hat{m}_{D} \Lambda_{e} / h}$.

Likewise consider the tunneling action in the ISS sector (ignoring the additional softterms for $\Phi$ when $n_{\Phi} \neq-1$ ). Defining $\varepsilon_{\text {hol }}=\sqrt{m_{D} / \Lambda_{\text {hol }}}$ and setting $h=1$, the VEV of the true supersymmetric minima in ISS is determined exactly:

$$
\Phi_{0}=\mu_{\mathrm{hol}} \varepsilon^{(2 F-3 N) / 2 N}
$$

where $\mu_{\text {hol }}^{2}=m_{D} \Lambda_{\text {hol }}$. An estimate for the tunnelling action that takes into account both the factor $e^{K}$ and the normalization of $\hat{\Phi}$ is then [39]

$$
S_{E} \sim 2 \pi^{2} N \varepsilon_{\mathrm{hol}}^{4(2 F-3 N) / N}\left(T_{2} U_{2}\right)^{1+3 n_{\Phi}} .
$$

Upon inspection, this expression is the only possible modular invariant combination with the correct functional dependence on $\varepsilon$ (and this could have been used as a short-cut to derive it). Indeed expressing holomorphic parameters in terms of physical ones, gives simply

$$
S_{E} \sim 2 \pi^{2} N \varepsilon^{4(2 F-3 N) / N}
$$

where $\varepsilon=\sqrt{\hat{m}_{D} / \Lambda_{e}}$.

\subsection{On $\Lambda_{e}>M_{K K}$}

An important point for the minimisation is that thanks to the remaining congruence subgroup symmetry there is no longer any reason to prevent $\Lambda_{e}>M_{K K}$. In particular the matching governed by eqs. (3.41)-(3.46) is still valid in these regions of parameter space as long as one bears in mind that the matching is between the effective $4 \mathrm{D}$ theories with KK modes integrated out. It is effectively being done at the scale $M_{K K}$. This fact will allow us to avoid the upper constraint in eq. (2.1).

Let us comment on this more explicitly. The picture of interest is where the original SQCD becomes strongly coupled at an energy scale $\Lambda_{e}>M_{K K}$, when it still contains many light KK modes. The effective $4 \mathrm{D}$ field theory description at this scale would resemble a truncated $6 \mathrm{D}$ theory, while the magnetic theory will be some unknown dual description. The physics of this full theory will be quite messy, so let us see what happens in a toymodel: motivated by the fact that the extra KK states in the spectrum of the electric theory include additional massive KK quarks with Dirac mass terms similar to those in eq. (3.41), as per section 2, consider including just these extra states as a set of $\Delta F$ flavours with mass $m_{\Delta F}$. One can "integrate in" these quarks to find a theory with dynamical scale $\Lambda_{\text {hol }}^{\prime}$ and beta function coefficient $b^{\prime}=b+\Delta F$. The scale $\Lambda_{\text {hol }}^{\prime}$ would then be regarded as the scale for the truncated $6 \mathrm{D}$ theory with its additional $\Delta F$ quarks, and its relation to $\Lambda_{\text {hol }}$ can be found by holomorphic matching at the scale $m_{\Delta F}$ :

$$
\left(\frac{\Lambda_{\mathrm{hol}}}{m_{\Delta F}}\right)^{b}=\left(\frac{\Lambda_{\mathrm{hol}}^{\prime}}{m_{\Delta F}}\right)^{b^{\prime}} .
$$

The magnetic equivalent of this situation is very well known: the $m_{\Delta F}$ operator gives rise to a linear meson term that via eq. (3.42) induces a Higgsing for the magnetic theory of 
$\langle q \cdot \tilde{q}\rangle=\hat{\Lambda} m_{\Delta F}$. Hence the "integrating in" of the electric theory, corresponds in the magnetic theory to an "unHiggsing" from $\mathrm{SU}(N)$ to $\mathrm{SU}(N+\Delta F)$, which gives a new beta function coefficient $\tilde{b}^{\prime}=\tilde{b}-2 \Delta F$, and an accompanying matching equation

$$
\left(\frac{\tilde{\Lambda}_{\mathrm{hol}}}{\sqrt{\hat{\Lambda} m_{\Delta F}}}\right)^{\tilde{b}}=\left(\frac{\tilde{\Lambda}_{\mathrm{hol}}^{\prime}}{\sqrt{\hat{\Lambda} m_{\Delta F}}}\right)^{\tilde{b}^{\prime}} .
$$

Now, upon inserting eqs. (3.68), (3.69), one finds that the 4D matching in eq. (3.45) derives from the matching equation of the full theory, namely

$$
\Lambda_{\text {hol }}^{\prime-b^{\prime}} \tilde{\Lambda}_{\text {hol }}^{\prime-\tilde{b}^{\prime}} \sim \hat{\Lambda}^{-(F+\Delta F)} .
$$

The point of this simple exercise is to demonstrate that no explicit powers of $T_{2} U_{2}$ can enter when one integrates out modes between $M_{K K}$ and $\Lambda_{e}$, because that would be in violation of the modular symmetry. In principle volume factors could have entered in a modular invariant way via the dependence on $\Delta F \sim\left(\Lambda_{e} / M_{K K}\right)^{d}$, but this would have introduced extra powers of $\Lambda$, and it would also have made the relation between the $6 \mathrm{D}$ and $4 \mathrm{D}$ dynamical scales singular in the decompactification limit. We conclude that the effective 4D relation in eq. (3.45) derives from the matching relation in the toy-model with all KK modes present in eq. (3.70), and neither version of the matching can contain factors of $T_{2}$ or $U_{2}$.

One does not expect that this conclusion would change if one were to start with the full $6 \mathrm{D}$ theory truncated at $\Lambda_{e}$, and its much more complicated magnetic dual (whatever form that may take). Thanks to the modular invariance, the "integrated in" $6 \mathrm{D}$ relation and the "integrated out" 4D relation are equally valid, although the $4 \mathrm{D}$ one is obviously the convenient choice. While it would be interesting to investigate how the $4 \mathrm{D}$ duality is embedded in the truncated $6 \mathrm{D}$ theory, knowledge of this is not required for the mechanism at hand. In particular, $\Lambda_{\text {hol }}$ is indeed just a parameter that specifies the dynamical scale of the effective $4 \mathrm{D}$ field theory when one integrates out all the KK physics, and $\tilde{\Lambda}_{\text {hol }}$ is the relevant dynamical scale for the $4 \mathrm{D}$ theory that emerges below $M_{K K}$, regardless of the relative size of $\Lambda_{\mathrm{hol}}$ and $M_{K K}$. Note that, if the couplings (i.e. $h=\Lambda_{\mathrm{hol}} / \hat{\Lambda}$ and friends) are of order unity, the dynamical scales of the truncated $6 \mathrm{D}$ theory are inevitably similar to those of the $4 \mathrm{D}$ theory regardless of the volume.

\section{Stabilisation in the string-embedded theories}

\subsection{Generic Casimir energy}

With all the necessary ingredients to hand, the minimisation can now be revisited. The generic case is treated in this subsection. The following subsection considers the UVCasimir case.

To start with, one can deduce from eq. (3.59) that there are always some mass-squareds of order $m_{3 / 2}^{2}$ in the infra-red of the ISS theory. It is convenient for the discussion in this and the following subsection to specialise to the weakly coupled case, and take $F \approx 3 \mathrm{~N} / 2$ 
giving $n_{q}, n_{\tilde{q}}, n_{\Phi} \approx-1 / 3$. This yields positive (physical) mass-squared operators of $\frac{2}{3} m_{3 / 2}^{2}$, regardless of $n_{Q}$ and $n_{\Lambda}$. Generalisation would be straightforward.

Therefore for the ISS mechanism to work as before (in particular for the rank-condition to be unchanged) one requires only that $\hat{\mu}^{2}=\hat{m}_{D} \Lambda_{e} / h \gtrsim \frac{2}{3} m_{3 / 2}^{2}$. There is now the additional attractive feature that gravity mediated contributions act to stabilise the smeson fields around the origin, quenching tunnelling completely. This means one is able to relax the conditions in eq. (2.1): one may work with $\Lambda_{e}>m_{3 / 2} \sim M_{K K}$ which then guarantees that $\hat{m}_{D}<\Lambda_{e}$ ensuring that the physical states all still remain in the ISS theory. It should be stressed that this does not cause a problem for the proper functioning of the ISS mechanism. As discussed in the previous section, the matching of the zero-mode SQCD theories can be done at the scale $M_{K K}$ and goes through as before regardless of the presence of heavier bulk modes. The magnetic ISS phase and the soft-terms all emerge below $M_{K K}$ with $\Lambda_{e}$ being the appropriate 4D SQCD scale, regardless of the relative size of $\Lambda_{e}$ and $M_{K K}$, and regardless of what additional states or physics might appear above the KK scale.

A possible generic difficulty with $\Lambda_{e}>m_{3 / 2}$ is rather that the ISS physics could change the original Casimir energy. One can see this sensitivity explicitly, by for example just removing the KK modes below the scale $\Lambda_{e}$ with an IR cut-off $\tau_{2}<1 / \Lambda_{e}^{2}$ on the Schwinger integral: this adds a term that dominates the contribution from the SQCD sector when $\Lambda_{e}>M_{K K}$. One can then see the advantage of the UV-Casimir theories whose cosmological constant is unchanged by such a cut-off: they automatically have a Casimir energy that is completely shielded from all IR physics. We take advantage of this feature in the next subsection. By contrast, for the generic case one must assume that the contribution to the cosmological constant from the ISS sector is swamped by the contribution from all the other massless degrees of freedom in the theory, that is $N_{f}^{(0)}-N_{b}^{(0)} \gg N_{f_{I S S}}^{(0)}-N_{b_{I S S}}^{(0)}$. Given the large number of states, this assumption is reasonable.

To perform the minimisation, let us consider the case $n_{Q}=-1$, which recall gives $n_{m_{D}}=+1$. (It is simple but not particularly instructive to generalise.) The physical Dirac mass then has the form $\hat{m}_{D}=\alpha_{D} \sqrt{4 T_{2} U_{2}}$ where $\alpha_{D}$ is a continuous parameter that must have weight +1 . (Therefore $\alpha_{D}$ represents an explicit breaking of the modular symmetry much like the Dirac mass in the original ISS scheme is an explicit breaking of the anomaly-free $R$-symmetry.) Note that $\alpha_{D}$ has mass dimension 2: henceforth all dimensionful quantities are in units of $M_{s}$. It will become clear that the above choice is consistent with the Dirac mass-term being a free parameter in the superymmetric theory.

Then using eq. (3.38) we have

$$
\begin{aligned}
V & =V_{C}+V_{I S S} \\
& =\frac{\pi^{3}}{120}\left(N_{f}^{0}-N_{b}^{0}\right) \frac{U_{2}^{3}}{T_{2}^{2}}+N \hat{m}_{D}^{2} \Lambda_{e}^{2}, \\
& =\frac{\pi^{3}}{120} \frac{\left(N_{f}^{0}-N_{b}^{0}\right)}{T_{2}^{2} \tilde{U}_{2}^{3}}+\frac{T_{2}}{\tilde{U}_{2}} 4 N \alpha_{D}^{2} e^{-\frac{16 \pi^{2}}{g_{s}^{2}|b|}+\frac{C}{b} \frac{\pi}{3}\left(T_{2}+\tilde{U}_{2}\right)} .
\end{aligned}
$$

Note that strictly speaking one should add the superpotential terms corresponding to the two sources of spontaneous supersymmetry breaking rather than the vacuum energies, and 
evaluate the resulting cosmological constant in the full supergravity theory. However the terms in the superpotential comprise a $U$ dependent part from the Casimir energy, and a $\Phi$ dependent part from the ISS contribution. The terms that are being neglected by not performing a full treatment can only arise from additional $U-\Phi$ mixing terms in the Kähler metric (since $F_{U}$ and $F_{\Phi}$ are the only non-zero $F$-terms); by flavour symmetry these have extra factors of $\langle\Phi\rangle$ which are zero at leading order.

The minimisation conditions give

$$
\tilde{U}_{2}=\frac{3}{2} T_{2}+\frac{15 b}{2 \pi C} .
$$

Assuming that the volume ends up at $T_{2} \gg 1$ (as will be verified in a moment), one may neglect the second term and use

$$
T_{2} U_{2}=\frac{2}{3}+\mathcal{O}\left(1 / T_{2}\right)
$$

and hence $M_{K K} \approx \sqrt{2 / 3} T_{2}^{-1}$. Note that $\hat{m}_{D} \approx \sqrt{8 / 3} \alpha_{D}$ regardless of the eventual scale of supersymmetry breaking. Therefore $\alpha_{D}$ can indeed be considered to be a parameter of the supersymmetric theory.

The potential becomes

$$
V\left(T_{2}\right)=\frac{\pi^{3}}{405} \frac{\left(N_{f}^{0}-N_{b}^{0}\right)}{T_{2}^{5}}+\frac{8 N \alpha_{D}^{2}}{3} e^{-\frac{16 \pi^{2}}{g_{s}^{2}|b|}+\frac{C}{b} \frac{5 \pi}{6} T_{2}} .
$$

The remaining one-dimensional minimisation can be done analogously to that in the $5 \mathrm{D}$ model of section 2. Using that notation, the fiducial scale of eq. (2.13) and the $T_{2}$ VEV are given by

$$
\begin{aligned}
\mu_{0}^{2} & \approx\left(\frac{5 \pi C}{6 b}\right)^{5} \frac{\pi^{3}}{6^{9}} \frac{\left(N_{f}^{0}-N_{b}^{0}\right)}{N \alpha_{D}^{2}} \\
& \approx \frac{4 \times 10^{-4}}{\alpha_{D}^{2}}\left(\frac{C}{b}\right)^{5} \frac{\left(N_{f}^{0}-N_{b}^{0}\right)}{N} \\
\frac{5 \pi C}{6 b} T_{2} & \approx \frac{16 \pi^{2}}{|b| g_{s}^{2}}+\ln \mu_{0}^{2},
\end{aligned}
$$

where, recall, the dynamical scale is then given by $\frac{\Lambda_{e}}{M_{K K}}=\mu_{0}$ (in string units). As mentioned above, with maximal SS phases, in order to avoid the SS soft-terms interfering with the ISS mechanism we choose $\Lambda_{e} \gtrsim M_{K K}$. From the above, assuming $\left(N_{f}^{0}-N_{b}^{0}\right) \sim N$ and $C \sim b$ requires $\alpha_{D}^{2} \ll 1$, which is consistent with $\hat{m}_{D} \ll M_{s}$. Indeed restoring the string scale we have

$$
\frac{\Lambda_{e}}{M_{K K}} \approx \sqrt{10^{-3}\left(\frac{C}{b}\right)^{5} \frac{\left(N_{f}^{0}-N_{b}^{0}\right)}{N}} \times \frac{M_{s}}{\hat{m}_{D}} .
$$

Summarising the $6 \mathrm{D}$ case then, when $g_{s} \ll 1$, the minimum is at

$$
T_{2} \approx \frac{2}{3 U_{2}} \approx \sqrt{\frac{2}{3}} M_{K K}^{-1} \approx \frac{96 \pi}{5|C| g_{s}^{2}},
$$

with $\Lambda_{e} \gtrsim M_{K K}$. As in the $5 \mathrm{D}$ case the potential rises exponentially fast beyond the minimum until $\Lambda_{e}$ surpasses $M_{s}$. A numerical example is shown in figure (4). 

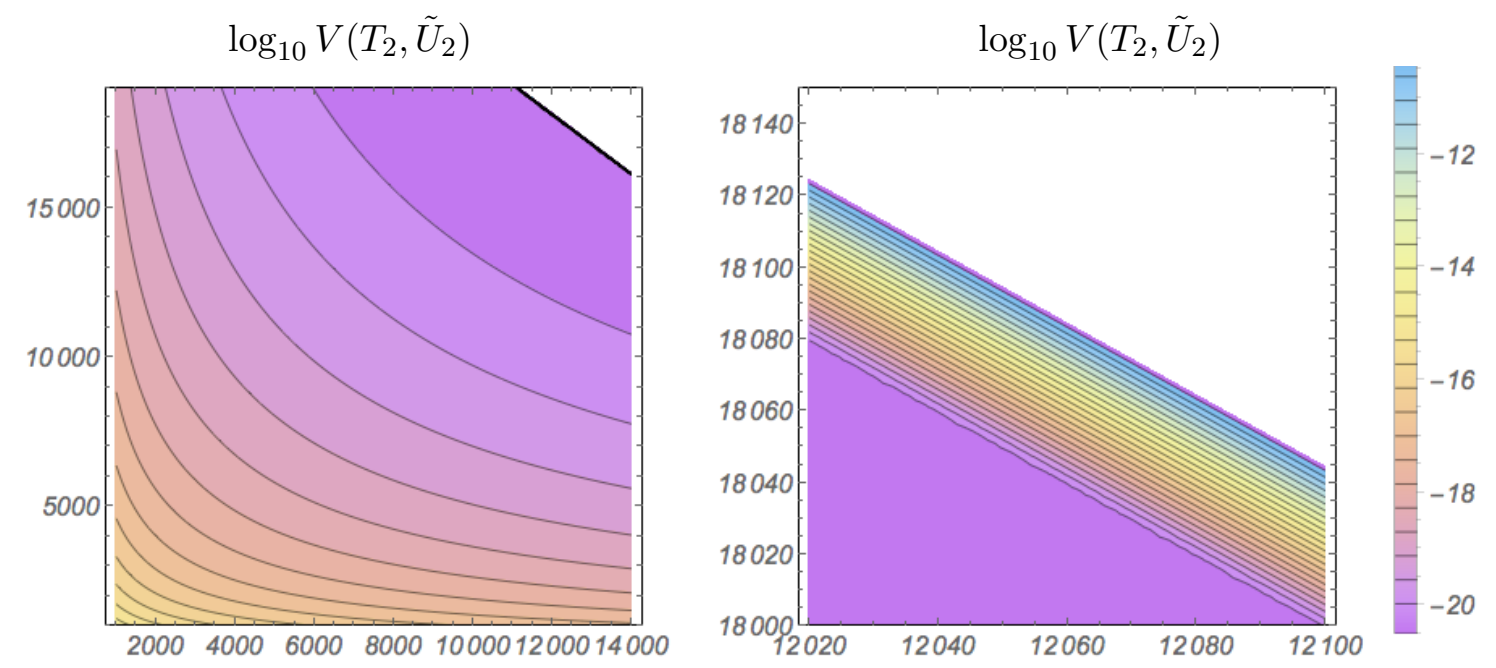

Figure 4. The potential for $b=-100, C=-50,\left(N_{f}^{0}-N_{b}^{0}\right)=N=10, \alpha_{D}=10^{-4}$ and $g_{s}=10^{-2}$. The approximation in eq. (4.5) gives a minimum at $T_{2}, \tilde{U}_{2}=12064,18096$ (in string units) respectively. As in the $5 \mathrm{D}$ case, the minimum lies close to a boundary in moduli space beyond which the effective field theory theory description breaks down as the dynamical QCD scale exceeds the string scale. The dynamical scale is $\Lambda_{e} \approx 35 M_{K K}$ but it can be easily brought closer to $M_{K K}$ with different choices of parameters, while $\Lambda_{e} \gg \hat{m}_{D}$ over the whole parameter space.

\subsection{UV-Casimir energy balanced against a gaugino condensate}

Next we consider the $N_{f}^{0}=N_{b}^{0}$ theories. As discussed earlier the Casimir energy in these cases is generated entirely by UV modes, so it is completely insensitive to the low energy physics. This separation is very interesting in the current context of balancing competing Scherk-Schwarz induced terms against non-perturbative IR physics, because it suggests that whatever mechanism is devised will be very robust. Moreover the two contributions to the cosmological constant may be consistently determined independently even though they necessarily involve the same moduli. In terms of the Schwinger integral, one can envisage the integrand as having two separate peaks, one at the stringy UV end and the other at the non-perturbative IR end. Therefore, one may simply add the two terms, which will be referred to as $V_{U V}$ and $V_{\mathrm{IR}}$, in the cosmological constant. Indeed $V_{U V}$ is computed in the string theory, while $V_{\mathrm{IR}}$ can be computed independently in the low energy effective field theory.

This opens up possibilities for stabilisation with non-perturbative physics that would otherwise be rather difficult to treat. For example gaugino condensation is now an attractive option for our IR physics rather than the ISS mechanism. Note that by contrast a standard SS Casimir energy (as considered in the previous section) balancing against a gaugino condensate would require a treatment of both terms simultaneously because they are functions only of $S, T, U$ and are not independent; essentially everything in that case would be happening in the IR, so it would be necessary to determine the full one-loop effective supergravity theory in order to compute the cosmological constant. 
To see this in practice, consider a single gaugino condensation contribution to $V_{\mathrm{IR}}$. The minimisation will now be done with all three fields, properly including the dynamics of the dilaton $S$ itself. However the philosophy is the same, namely we expect to end up in a stable or metastable minimum that has relatively large $S$ compensated by relatively large $T$ and $\tilde{U}$.

The IR contribution to the potential is calculated in supergravity, incorporating the superpotential $W_{\mathrm{SS}}$ in eq. (3.16) for the Scherk-Schwarz background, in addition to the gaugino condensate, that is $W_{\mathrm{IR}}=W_{\mathrm{SS}}+W_{\mathrm{gc}}$. The latter is described by the well-known superpotential

$$
W_{\mathrm{gc}}=d \Lambda_{\mathrm{hol}}^{3},
$$

where $d$ is a constant, and now

$$
\Lambda_{\mathrm{hol}} \approx e^{-\frac{8 \pi^{2}}{|b|} S+\frac{C}{b} \frac{\pi}{6}(T+\tilde{U})}
$$

is the holomorphic scale for the pure Yang-Mills theory. Eq. (3.31) with $F=0$ and $b_{0}=-3 N$ correctly gives $n_{W_{\mathrm{gc}}}=-1$. The approximation refers to $T_{2}, \tilde{U}_{2} \gg 1$ near $i U=1$ and as discussed earlier it breaks the modular symmetries. In the Scherk-Schwarz background, adding $W_{\mathrm{SS}}$ then incorporates the effect of the shifted mass spectrum. We know that the potential without $W_{\mathrm{gc}}$ is entirely flat so one can anticipate that the resulting contribution involves powers of $W_{\mathrm{gc}}$.

Some care is required regarding phases: bearing in mind the cosmological constant discussion in section 3.2, one can anticipate that $U_{1}$ and $T_{1}$ will ultimately be fixed to zero by $V_{U V}$, and therefore one does not need to consider them further. However the phase of the dilaton $S_{1}$ remains as a free field that is fixed by the gaugino condensate.

Using eq. (3.40), the potential is conveniently arranged (at $U_{1}=T_{1}=0$ ) as

$$
\begin{aligned}
V=V_{U V}+V_{\mathrm{IR}}= & 2\left(N_{f}^{1}-N_{b}^{1}\right) T_{2}^{-3 / 4} \tilde{U}_{2}^{-7 / 4} e^{-2 \pi \sqrt{T_{2} \tilde{U}_{2}}}+B\left(\left|\Lambda_{\text {hol }}^{3}\right|-\frac{A}{B}\right)^{2}-\frac{|A|^{2}}{B} \\
A \frac{S_{2} T_{2}}{d}= & \frac{1}{2 \sqrt{2}}\left(1+\log \left|\Lambda_{\text {hol }}^{3}\right|\right) \\
B \frac{S_{2} T_{2}}{d^{2} \tilde{U}_{2}}= & \frac{1}{2} \log \mid \Lambda_{\text {hol }}^{3}\left(\log \left|\Lambda_{\text {hol }}^{3}\right|-1\right)-\frac{\pi}{2} \frac{C}{b}\left(\left(T_{2}+\tilde{U}_{2}\right) \log \left|\Lambda_{\text {hol }}^{3}\right|-\tilde{U}_{2}\right) \\
& +\left(\frac{\pi}{2} \frac{C}{b}\right)^{2}\left(T_{2}^{2}+T_{2} \tilde{U}_{2}+\tilde{U}_{2}^{2}\right) .
\end{aligned}
$$

The entire $S_{2}$ dependence is contained within the $e^{K}$ prefactors and the $\left|\Lambda_{\text {hol }}\right|$ dependence, while $S_{1}$ simply adjusts the phase of $\Lambda_{\text {hol }}$ so that it comes to rest where it minimises the square with a relative minus sign as shown. The minimisation with respect to the dilaton is then dominated by the complete square term, which gives the approximation

$$
\begin{aligned}
\left|\Lambda_{\mathrm{hol}}\right| & \approx A / B\left(1+\mathcal{O}\left(24 \pi^{2} S_{2} /|b|\right)\right) \\
& \approx \frac{1}{2 \sqrt{2} d}\left(\frac{\pi}{2} \frac{C}{b}\right)^{-2} \frac{1}{\tilde{U}_{2}\left(T_{2}^{2}+T_{2} \tilde{U}_{2}+\tilde{U}_{2}^{2}\right)} .
\end{aligned}
$$


The error on the right hand side of this equation is due to the $e^{K}$ pre-factor and is negligible when the gauge coupling at the string scale ends up being weak (as is the case of interest). The $A / B$ term on the right hand side depends only logarithmically on $\Lambda_{\text {hol }}$; the approximation can be improved by iteration if required but as long as the volume $T_{2}$ is large, the zeroth order expression shown on the second line is sufficiently accurate.

The potential is qualitatively different from that in the ISS case because the single gaugino condensate does not by itself give a minimum in $T_{2}$ or $U_{2}$. In fact without the $V_{U V}$ contribution the potential has a runaway to small moduli (where our approximations break down) or to infinity. With $V_{U V}$ however a minimum is found where the two terms $V_{U V}$ and $V_{\mathrm{IR}}$ balance, giving rise to the novel phenomenon that the non-perturbative lowenergy contribution self-tunes to be of the same order as the exponentially suppressed UVCasimir energy. A framework in which an exponentially small UV cosmological constant governs and stabilises non-perturbative IR physics without being disrupted itself seems of general interest.

An example potential is shown in figure 5 for a typical set of parameters. In addition the plot shows the line where $V_{U V}=V_{\mathrm{IR}}$ close to the actual minimum. The nett result is a minimum in which all the moduli are stabilised and $\Lambda_{\text {hol }} \sim M_{s} / 10$. Notice that the approximation $T_{2} \approx \frac{2}{3} \tilde{U}_{2}$ at the minimum still holds. This example takes $N_{f}^{1}-N_{b}^{1}=10^{6}$ which may seem large, but one should recall that there are very many excitations at the first string excitation level, and in fact this number is quite typical. Not surprisingly, reducing this number (and increasing $d$ ) moves the minimum closer to the origin, where neglected contributions to $V_{U V}$ such as those from winding modes will start to become important. Discussion of this effect along with explicit examples was presented recently in ref. [63], and it would be interesting to incorporate these additional terms in detail.

\section{Conclusion}

In summary, it is argued that a general means of addressing the decompactification problem dynamically is to balance non-perturbative physics contributions to the vacuum energy against the Casimir energy in Scherk-Schwarzed theories. Due to universality in both the threshold corrections and the gauge couplings, the stable minimum will have consistently large (order one) gauge couplings for any gauge group that shares the same $\mathcal{N}=2$ beta function for bulk modes as the gauge group taking part in the minimisation. By contrast gauge symmetries with the wrong-sign beta function will remain as effectively global symmetries.

Both the ISS mechanism and a single gaugino condensate were considered for the stabilising non-perturbative physics in the case of compactification from $6 \mathrm{D}$ to $4 \mathrm{D}$ in heterotic strings. In either case, both the Scherk-Schwarz contribution and the non-perturbative contribution to supersymmetry breaking can be written as superpotential terms in $\mathcal{N}=1$ theories, which spontaneously break supersymmetry.

The ISS mechanism is interesting because it gives novel cross-checks based on the residual modular symmetry of the theory, and also allows one to handle the supersymmetry breaking from the ISS mechanism and the Scherk-Schwarz breaking simultaneously. By 


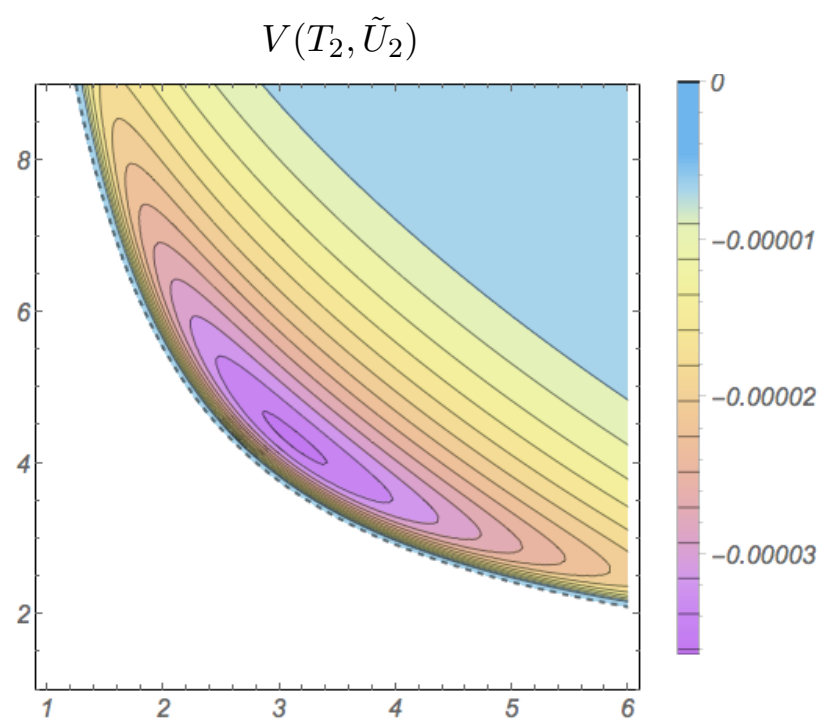

Figure 5. The potential for $b=-80, C=-20,\left(N_{f}^{1}-N_{b}^{1}\right)=10^{6}$ with $d=0.1$. The dashed line marks where the IR contribution to the potential is equal to the UV one. In practice the prefactors make very little difference to the qualitative form of the potential, but move the minimum along $T_{2} \approx \frac{2}{3} \tilde{U}_{2}$.

contrast the gaugino condensate is interesting when the original Scherk-Schwarzed theory retains Bose-Fermi degeneracy and has exponentially suppressed cosmological constant. An important aspect of the SS induced cosmological constant in this case is that it is entirely generated by heavy modes and as such is completely immune to any non-perturbative physics that might be added in the IR to provide a balancing contribution. It allows very simple treatment of the minimisation which in this case takes place at moderate volume. A full treatment in this generic set-up (that is, including the stabilisation of the compactification moduli as well as the original dilaton) was presented. The energetic separation between competing and balancing UV and IR induced terms in the potential makes stabilisation very robust, and seems to be something that has not been remarked upon before. It would be of interest to apply the mechanism to explicit examples, such as the models discussed recently in ref. [63], which has some intriguing overlaps with the work described here.

\section{Acknowledgments}

I would like to thank Keith Dienes, Costas Kounnas, Alberto Mariotti, Eirini Mavroudi and Carlos Tamarit for discussions.

\section{A Summary of $\operatorname{SL}(2, \mathbb{Z})_{U}$ and $\operatorname{SL}(2, \mathbb{Z})_{U}$ structure}

The heterotic modular symmetries begin life as subgroups of the exact $O(16+d, d, \mathbb{Z})$ target-space automorphisms of the Narain lattice [60-62]. The $T$-modular transformations are presented here for reference. They form a subgroup $\operatorname{SL}(2, \mathbb{Z})_{T}$, under which the fields 
transform as

$$
\begin{aligned}
T & \rightarrow \frac{a T-i b}{i c T+d}, \\
U & \rightarrow U-i c \frac{\phi \phi^{\prime}}{i c T+d}, \\
S & \rightarrow S-\delta_{G S} \log (i c T+d), \\
\phi, \phi^{\prime} & \rightarrow \frac{\phi, \phi^{\prime}}{i c T+d},
\end{aligned}
$$

with $a, b, c, d \in \mathbb{Z}$ and $a d-b c=1$, while the $U$-modular transformation $\operatorname{SL}(2, \mathbb{Z})_{U}$ is

$$
\begin{aligned}
U & \rightarrow \frac{a U-i b}{i c U+d}, \\
T & \rightarrow T-i c \frac{\phi \phi^{\prime}}{i c U+d}, \\
S & \rightarrow S-\delta_{G S} \log (i c U+d), \\
\phi, \phi^{\prime} & \rightarrow \frac{\phi, \phi^{\prime}}{i c U+d} .
\end{aligned}
$$

Some useful identities under the $i T \rightarrow-1 / i T$ transformation of the $\operatorname{SL}(2, \mathbb{Z})_{T}$ modular group for example, are

$$
\begin{aligned}
T+\bar{T} & \rightarrow \frac{T+\bar{T}}{|i c T+d|^{2}} \\
\eta(i T)^{2} & \rightarrow(i c T+d) \eta(i T)^{2} \\
|\eta(i T)|^{4}(T+\bar{T}) & \rightarrow|\eta(i T)|^{4}(T+\bar{T}),
\end{aligned}
$$

so that the Kähler potential $K=-\log \left(4 T_{2} U_{2}-\left|\phi+\bar{\phi}^{\prime}\right|^{2}\right)$ transforms as $K \rightarrow K+$ $\log |i c T+d|^{2}$. Thus the superpotential has to have weight -1 under $\operatorname{SL}(2, \mathbb{Z})_{T, U}$.

Open Access. This article is distributed under the terms of the Creative Commons Attribution License (CC-BY 4.0), which permits any use, distribution and reproduction in any medium, provided the original author(s) and source are credited.

\section{References}

[1] J. Scherk and J.H. Schwarz, Spontaneous Breaking of Supersymmetry Through Dimensional Reduction, Phys. Lett. 82B (1979) 60 [INSPIRE].

[2] J. Scherk and J.H. Schwarz, How to Get Masses from Extra Dimensions, Nucl. Phys. B 153 (1979) 61 [INSPIRE].

[3] I. Antoniadis, A possible new dimension at a few TeV, Phys. Lett. B 246 (1990) 377 [INSPIRE].

[4] I. Antoniadis, K.S. Narain and T.R. Taylor, Higher genus string corrections to gauge couplings, Phys. Lett. B 267 (1991) 37 [INSPIRE].

[5] I. Antoniadis, S. Dimopoulos, A. Pomarol and M. Quirós, Soft masses in theories with supersymmetry breaking by TeV compactification, Nucl. Phys. B 544 (1999) 503 [hep-ph/9810410] [INSPIRE]. 
[6] A. Delgado, A. Pomarol and M. Quirós, Supersymmetry and electroweak breaking from extra dimensions at the TeV scale, Phys. Rev. D 60 (1999) 095008 [hep-ph/9812489] [INSPIRE].

[7] D.M. Ghilencea, H.P. Nilles and S. Stieberger, Divergences in Kaluza-Klein models and their string regularization, New J. Phys. 4 (2002) 15 [hep-th/0108183] [INSPIRE].

[8] S. Dimopoulos, K. Howe and J. March-Russell, Maximally Natural Supersymmetry, Phys. Rev. Lett. 113 (2014) 111802 [arXiv: 1404.7554] [INSPIRE].

[9] T. Cohen, N. Craig, H.K. Lou and D. Pinner, Folded Supersymmetry with a Twist, JHEP 03 (2016) 196 [arXiv: 1508.05396] [InSPIRE].

[10] I. Garcia Garcia, K. Howe and J. March-Russell, Natural Scherk-Schwarz Theories of the Weak Scale, JHEP 12 (2015) 005 [arXiv:1510.07045] [INSPIRE].

[11] K. Tobioka, R. Kitano and H. Murayama, Enhanced Higgs Mass in Compact Supersymmetry, JHEP 04 (2016) 025 [arXiv: 1511.04081] [InSPIRE].

[12] I. Antoniadis, K. Benakli and M. Quirós, Sequestered gravity in gauge mediation, Eur. Phys. J. C 76 (2016) 363 [arXiv:1512.00029] [InSPIRE].

[13] M. Reece and W. Xue, SUSY's Ladder: reframing sequestering at Large Volume, JHEP 04 (2016) 045 [arXiv: 1512.04941] [INSPIRE].

[14] A. Delgado, M. Garcia-Pepin, G. Nardini and M. Quirós, Natural Supersymmetry from Extra Dimensions, arXiv:1608.06470 [INSPIRE].

[15] S. Abel, K.R. Dienes and E. Mavroudi, Towards a nonsupersymmetric string phenomenology, Phys. Rev. D 91 (2015) 126014 [arXiv:1502.03087] [InSPIRE].

[16] E. Ponton and E. Poppitz, Casimir energy and radius stabilization in five-dimensional orbifolds and six-dimensional orbifolds, JHEP 06 (2001) 019 [hep-ph/0105021] [INSPIRE].

[17] M. Borunda, M. Serone and M. Trapletti, On the quantum stability of IIB orbifolds and orientifolds with Scherk-Schwarz SUSY breaking, Nucl. Phys. B 653 (2003) 85 [hep-th/0210075] [INSPIRE].

[18] G. von Gersdorff, M. Quirós and A. Riotto, Scherk-Schwarz supersymmetry breaking with radion stabilization, Nucl. Phys. B 689 (2004) 76 [hep-th/0310190] [INSPIRE].

[19] E. Dudas and C. Timirgaziu, Nontachyonic Scherk-Schwarz compactifications, cosmology and moduli stabilization, JHEP 03 (2004) 060 [hep-th/0401201] [INSPIRE].

[20] E. Dudas and M. Quirós, Five-dimensional massive vector fields and radion stabilization, Nucl. Phys. B 721 (2005) 309 [hep-th/0503157] [INSPIRE].

[21] G. von Gersdorff and A. Hebecker, Radius stabilization by two-loop Casimir energy, Nucl. Phys. B 720 (2005) 211 [hep-th/0504002] [INSPIRE].

[22] C. Angelantonj, M. Cardella and N. Irges, An Alternative for Moduli Stabilisation, Phys. Lett. B 641 (2006) 474 [hep-th/0608022] [INSPIRE].

[23] A.P. Braun, A. Hebecker and M. Trapletti, Moduli stabilization in (string) model building: Gauge fluxes and loops, arXiv:0710.4877 [INSPIRE].

[24] C. Gross and A. Hebecker, A Realistic Unified Gauge Coupling from the Micro-Landscape of Orbifold GUTs, Nucl. Phys. B 821 (2009) 354 [arXiv:0812.4267] [InSPIRE].

[25] K.R. Dienes, E. Dudas and T. Gherghetta, Extra space-time dimensions and unification, Phys. Lett. B 436 (1998) 55 [hep-ph/9803466] [INSPIRE]. 
[26] K.R. Dienes, E. Dudas and T. Gherghetta, Grand unification at intermediate mass scales through extra dimensions, Nucl. Phys. B 537 (1999) 47 [hep-ph/9806292] [INSPIRE].

[27] K.R. Dienes, E. Dudas and T. Gherghetta, TeV scale GUTs, hep-ph/9807522 [INSPIRE].

[28] K.R. Dienes, E. Dudas and T. Gherghetta, GUT precursors and nontrivial fixed points in higher dimensional gauge theories, Phys. Rev. Lett. 91 (2003) 061601 [hep-th/0210294] [INSPIRE].

[29] K.R. Dienes, E. Dudas and T. Gherghetta, GUT precursors and fixed points in higher-dimensional theories, Pramana 62 (2004) 219.

[30] E. Caceres, V.S. Kaplunovsky and I.M. Mandelberg, Large volume string compactifications, revisited, Nucl. Phys. B 493 (1997) 73 [hep-th/9606036] [InSPIRE].

[31] E. Kiritsis, C. Kounnas, P.M. Petropoulos and J. Rizos, Solving the decompactification problem in string theory, Phys. Lett. B 385 (1996) 87 [hep-th/9606087] [INSPIRE].

[32] E. Kiritsis, C. Kounnas, P.M. Petropoulos and J. Rizos, String threshold corrections in models with spontaneously broken supersymmetry, Nucl. Phys. B 540 (1999) 87 [hep-th/9807067] [INSPIRE].

[33] I. Antoniadis and K. Benakli, Large dimensions and string physics in future colliders, Int. J. Mod. Phys. A 15 (2000) 4237 [hep-ph/0007226] [INSPIRE].

[34] A.E. Faraggi, C. Kounnas and H. Partouche, Large volume SUSY breaking with a solution to the decompactification problem, Nucl. Phys. B 899 (2015) 328 [arXiv:1410.6147] [INSPIRE].

[35] C. Kounnas and H. Partouche, Stringy $N=1$ super no-scale models, PoS (PLANCK 2015) 070 [arXiv: 1511.02709] [INSPIRE].

[36] C. Kounnas and H. Partouche, Super no-scale models in string theory, Nucl. Phys. B 913 (2016) 593 [arXiv : 1607.01767] [INSPIRE].

[37] H. Partouche, Large volume supersymmetry breaking without decompactification problem, arXiv: 1601.04564 [INSPIRE].

[38] C. Angelantonj, I. Florakis and M. Tsulaia, Generalised universality of gauge thresholds in heterotic vacua with and without supersymmetry, Nucl. Phys. B 900 (2015) 170 [arXiv: 1509.00027] [INSPIRE].

[39] K.A. Intriligator, N. Seiberg and D. Shih, Dynamical SUSY breaking in meta-stable vacua, JHEP 04 (2006) 021 [hep-th/0602239] [INSPIRE].

[40] R. Rohm, Spontaneous Supersymmetry Breaking in Supersymmetric String Theories, Nucl. Phys. B 237 (1984) 553 [inSPIRE].

[41] S. Ferrara, C. Kounnas and M. Porrati, $N=1$ Superstrings With Spontaneously Broken Symmetries, Phys. Lett. B 206 (1988) 25 [INSPIRE].

[42] S. Ferrara, C. Kounnas and M. Porrati, Superstring Solutions With Spontaneously Broken Four-dimensional Supersymmetry, Nucl. Phys. B 304 (1988) 500 [INSPIRE].

[43] S. Ferrara, C. Kounnas, M. Porrati and F. Zwirner, Superstrings with Spontaneously Broken Supersymmetry and their Effective Theories, Nucl. Phys. B 318 (1989) 75 [InSPIRE].

[44] C. Kounnas and B. Rostand, Coordinate Dependent Compactifications and Discrete Symmetries, Nucl. Phys. B 341 (1990) 641 [INSPIRE]. 
[45] E. Kiritsis and C. Kounnas, Perturbative and nonperturbative partial supersymmetry breaking: $N=4 \rightarrow N=2 \rightarrow N=1$, Nucl. Phys. B 503 (1997) 117 [hep-th/9703059] [INSPIRE].

[46] E. Dudas and J. Mourad, Brane solutions in strings with broken supersymmetry and dilaton tadpoles, Phys. Lett. B 486 (2000) 172 [hep-th/0004165] [INSPIRE].

[47] C.A. Scrucca and M. Serone, On string models with Scherk-Schwarz supersymmetry breaking, JHEP 10 (2001) 017 [hep-th/0107159] [INSPIRE].

[48] M.J. Duncan and L.G. Jensen, Exact tunneling solutions in scalar field theory, Phys. Lett. B 291 (1992) 109 [INSPIRE].

[49] A. Riotto and E. Roulet, Vacuum decay along supersymmetric flat directions, Phys. Lett. B 377 (1996) 60 [hep-ph/9512401] [INSPIRE].

[50] M.A. Luty and R. Rattazzi, Soft supersymmetry breaking in deformed moduli spaces, conformal theories and $N=2$ Yang-Mills theory, JHEP 11 (1999) 001 [hep-th/9908085] [INSPIRE].

[51] S. Abel, M. Buican and Z. Komargodski, Mapping Anomalous Currents in Supersymmetric Dualities, Phys. Rev. D 84 (2011) 045005 [arXiv:1105.2885] [InSPIRE].

[52] V.S. Kaplunovsky, One Loop Threshold Effects in String Unification, Nucl. Phys. B 307 (1988) 145 [Erratum ibid. B 382 (1992) 436] [hep-th/9205068] [INSPIRE].

[53] V. Kaplunovsky and J. Louis, On gauge couplings in string theory, Nucl. Phys. B 444 (1995) 191 [hep-th/9502077] [INSPIRE].

[54] Z. Lalak, S. Lavignac and H.P. Nilles, String dualities in the presence of anomalous U(1) symmetries, Nucl. Phys. B 559 (1999) 48 [hep-th/9903160] [InSPIRE].

[55] S.L. Parameswaran, S. Ramos-Sanchez and I. Zavala, On Moduli Stabilisation and de Sitter Vacua in MSSM Heterotic Orbifolds, JHEP 01 (2011) 071 [arXiv: 1009.3931] [INSPIRE].

[56] L.J. Dixon, V. Kaplunovsky and J. Louis, Moduli dependence of string loop corrections to gauge coupling constants, Nucl. Phys. B 355 (1991) 649 [INSPIRE].

[57] P. Mayr and S. Stieberger, Threshold corrections to gauge couplings in orbifold compactifications, Nucl. Phys. B 407 (1993) 725 [hep-th/9303017] [INSPIRE].

[58] E. Kiritsis and C. Kounnas, Infrared regularization of superstring theory and the one loop calculation of coupling constants, Nucl. Phys. B 442 (1995) 472 [hep-th/9501020] [INSPIRE].

[59] C. Angelantonj, I. Florakis and B. Pioline, Rankin-Selberg methods for closed strings on orbifolds, JHEP 07 (2013) 181 [arXiv:1304.4271] [INSPIRE].

[60] A. Giveon, M. Porrati and E. Rabinovici, Target space duality in string theory, Phys. Rept. 244 (1994) 77 [hep-th/9401139] [inSPIRE].

[61] G. Lopes Cardoso, D. Lüst and T. Mohaupt, Moduli spaces and target space duality symmetries in $(0,2) Z(N)$ orbifold theories with continuous Wilson lines, Nucl. Phys. B 432 (1994) 68 [hep-th/9405002] [INSPIRE].

[62] I. Antoniadis, E. Gava, K.S. Narain and T.R. Taylor, Effective $\mu$-term in superstring theory, Nucl. Phys. B 432 (1994) 187 [hep-th/9405024] [INSPIRE].

[63] I. Florakis and J. Rizos, Chiral Heterotic Strings with Positive Cosmological Constant, Nucl. Phys. B 913 (2016) 495 [arXiv: 1608.04582] [InSPIRE]. 